\title{
Assessing macro uncertainty in real-time when data are subject to revision
}

Article

Accepted Version

Clements, M. P. (2017) Assessing macro uncertainty in realtime when data are subject to revision. Journal of Business \& Economic Statistics, 35 (3). pp. 420-433. ISSN 0735-0015 doi: https://doi.org/10.1080/07350015.2015.1081596 Available at https://centaur.reading.ac.uk/41466/

It is advisable to refer to the publisher's version if you intend to cite from the work. See Guidance on citing.

To link to this article DOI: http://dx.doi.org/10.1080/07350015.2015.1081596

Publisher: Taylor \& Francis

All outputs in CentAUR are protected by Intellectual Property Rights law, including copyright law. Copyright and IPR is retained by the creators or other copyright holders. Terms and conditions for use of this material are defined in the End User Agreement.

\section{www.reading.ac.uk/centaur}

\section{CentAUR}

Central Archive at the University of Reading

Reading's research outputs online 


\title{
Assessing Macro Uncertainty In Real-Time When Data Are Subject To Revision
}

\author{
Michael P. Clements* \\ ICMA Centre \\ Henley Business School \\ University of Reading \\ m.p.clements@reading.ac.uk
}

August 6, 2015

\begin{abstract}
Model-based estimates of future uncertainty are generally based on the in-sample fit of the model, as when Box-Jenkins prediction intervals are calculated. However, this approach will generate biased uncertainty estimates in real time when there are data revisions. A simple remedy is suggested, and used to generate more accurate prediction intervals for 25 macroeconomic variables, in line with the theory. A simulation study based on an empirically-estimated model of data revisions for US output growth is used to investigate small-sample properties.
\end{abstract}

Keywords: in-sample uncertainty, out-of-sample uncertainty, real-time-vintage estimation

JEL code: C53.

*Michael P. Clements is an Associate member of the Institute for New Economic Thinking, Oxford Martin School, University of Oxford, UK. 


\section{Assessing Macro Uncertainty In Real-Time \\ When Data Are Subject To Revision}

Model-based estimates of future uncertainty are generally based on the in-sample fit of the model, as when Box-Jenkins prediction intervals are calculated. However, this approach will generate biased uncertainty estimates in real time when there are data revisions. A simple remedy is suggested, and used to generate more accurate prediction intervals for 25 macroeconomic variables, in line with the theory. A simulation study based on an empirically-estimated model of data revisions for US output growth is used to investigate small-sample properties.

Keywords: in-sample uncertainty, out-of-sample uncertainty, real-time-vintage estimation

JEL code: C53. 


\section{Introduction}

There has been much recent interest in macro-forecasting in real-time. By this we mean how the forecasting model should be specified, estimated, and the resulting forecasts evaluated, once we acknowledge that the data on which these three activities is based is subject to revision (for all but a small number of series such as interest rates and exchange rates). A number of papers have considered modelling the revisions process (see, e.g., Cunningham, Eklund, Jeffery, Kapetanios and Labhard (2009), Jacobs and van Norden (2011), Kishor and Koenig (2012)); or using single-equation models with 'real-time-vintages' (as in Koenig, Dolmas and Piger (2003), Clements and Galvão (2013b)) which we refer to as RTV-estimation and discuss below; or modelling multiple vintages of data, as with vintage-based vector autoregressive models (see, e.g., Patterson (1995, 2003), Clements and Galvão (2013a)). Other papers have considered whether assessing predictability in real-time may change the conclusions one would draw concerning putative explanatory forces, or the usefulness of estimates of the output gap as a guide to monetary policy in real time. On the former, it has been argued that the use of final-revised data may exaggerate the predictive power of explanatory variables relative to what could actually have been achieved at the time using the then available data (see, e.g., Robertson and Tallman (1998), Faust, Rogers and Wright (2003)), and on the latter see Orphanides (2001), Orphanides and van Norden (2005), Garratt, Lee, Mise and Shields (2009, 2008) and Clements and Galvão (2012), inter alia. Croushore (2011a, 2011b) provide useful state-of-the-art reviews.

These related strands of research are all concerned with first-moment prediction: either improving the accuracy of forecasts of the conditional expectation, or of providing a more realistic appraisal of the accuracy of these predictions. In this paper we instead investigate the implications of data revisions for assessments of forecast uncertainty, specifically, the accuracy of prediction intervals. We show that the 'traditional' approaches to calculating prediction intervals will tend to be either too wide, when data revisions 'add news', or too narrow, when the revisions process 'removes noise'. The standard approach is due to Box-Jenkins (Box and Jenkins (1970)). The effects of data vintages in real-time analyses are first-order, in the sense that they do not disappear when the sample size gets large, and are not caused by non-normal errors. It is recognized in the literature that Box-Jenkins prediction intervals suffer from the neglect of parameter estimation uncertainty and the possible non-normality of the underlying 
model's disturbances, and as a result there has been much interest in bootstrapping prediction intervals (see e.g., Thombs and Schucany (1990)). Yet we show that in real-time prediction intervals will have incorrect coverage even in the absence of these problems (i.e., in the absence of parameter estimation uncertainty, and when the disturbances are normal), because of data vintage effects. We use simple Box-Jenkins intervals to focus on data vintage effects.

Clearly, the effects of data vintages on first and second-moment prediction can be sidestepped by considering fully-revised data, whence observations are no longer subject to revision. Of course any observation no matter how far back in time may be changed in response to farreaching methodological changes. By fully-revised data we mean data that have undergone the initial and three annual rounds of revisions (see e.g., Landefeld, Seskin and Fraumeni (2008) for a description of the revisions process of the US Bureau of Economic Analysis NIPA data). Pseudo out-of-sample exercises use fully-revised data (e.g., the vintage of data available at the time of the study), and at each point in time the forecasting models are specified and the parameters estimated using only data for time periods up to that point in time. Such exercises are useful as a way of assessing how well the model or models fit or forecast the true data, and the out-of-sample aspect guards against 'overfitting', i.e., the models capturing chance or non-recurrent sample-specific features. A real-time forecasting exercise mimics the environment a real-world forecaster faces - at each point in time the forecasting models are specified and the parameters estimated using only data for time periods up to that point in time, but in addition the data are taken from the vintages that would have been available at that point in time. Real-time exercises are required to provide fair assessments of the relative accuracy of the model forecasts compared to survey expectations, for example.

To illustrate, assume a one period delay in data availability. Then at time $t$ a forecaster will have access to the vintage- $t$ value of the period $t-1$ observation, denoted $y_{t-1}^{t}$, and similarly the second estimate of the $t-2$ period, $y_{t-2}^{t}$, and so on. If the forecaster wishes to use only data which has been revised $n$ times, say, the most recent data that could be used would be for $t-n-1$ (i.e., $y_{t-n-1}^{t}, y_{t-n-2}^{t}, \ldots$ ). It will rarely be optimal to ignore data for periods $t-n-2, \ldots, t-1$ (for large $n$ ), so the real-world forecaster will be forced to work with data subject to revision. This is the environment we seek to mimic in the real-time analysis.

Section 2 presents a simple example to motivate the concerns of the paper: the true model 
is a zero-mean first-order autoregressive process $(\mathrm{AR}(1))$, and the true value of the process is revealed the period after the first estimate, so only the first estimate is subject to revision. Further, we ignore estimation uncertainty, so that model parameters take on their population parameters. We show that the standard approach gives an incorrect assessment of forecast uncertainty, and consequently incorrectly-sized prediction intervals. We focus on prediction intervals, but the points we make apply more generally to measures of forecast uncertainty (such as forecast densities). In section 3 we broaden the analysis of section 2 to an $\operatorname{AR}(p)$ for the true process, and allow a general revisions process with multiple revisions. As in the simple case, there will in general be a misleading assessment of the uncertainty surrounding future outcomes. We then suggest a remedy that has been used for first-moment prediction, and has the virtue of simplicity and does not require that the revisions process be modelled: section 4. Section 5 supposes the goal is to estimate the uncertainty about future revised values, as opposed to first-release data. Section 6 provides an empirical illustration, and shows the improvements that result from using the approach advocated in this paper. We consider 25 macro variables, which exhibit different patterns of revisions, so that the conclusions we draw are reasonably general and do not rest on a few variables with (possibly) idiosyncratic features. In section 7 we present a simulation study of the two approaches to calculating prediction intervals in a controlled environment that abstracts from various factors that might affect the empirical comparisons, such as parameter non-constancies, for example. Finally, section 8 offers some concluding remarks.

\section{Motivating example}

Suppose the true (i.e., fully-revised) values $y_{t}$ follow an $\operatorname{AR}(1)$ :

$$
y_{t}=\alpha y_{t-1}+\eta_{t}+v_{t}
$$

and the estimates of $y_{t}$ are given by:

$$
\begin{array}{rrr}
y_{t}^{t+1} & = & y_{t}-v_{t}+\varepsilon_{t} \\
y_{t}^{t+n} & = & y_{t}
\end{array}
$$


for $n=2,3, \ldots$. We assume $\eta_{t}, v_{t}$ and $\varepsilon_{t}$ are mutually uncorrelated, zero-mean iid random variables. Then the revision $y_{t}^{t+2}-y_{t}^{t+1}=v_{t}-\varepsilon_{t}$ consists of a noise component (when $\sigma_{\varepsilon}^{2}=$ $E\left(\varepsilon_{t}^{2}\right) \neq 0$ ) and a news component (when $\sigma_{v}^{2}=E\left(v_{t}^{2}\right) \neq 0$ ). The news/noise characterization of data revisions is due to Mankiw and Shapiro (1986). Suppose that revisions are purely news, so that $\sigma_{\varepsilon}^{2}=0$. Then the first estimate $y_{t}^{t+1}=\alpha y_{t-1}+\eta_{t}$ does not contain the news component $v_{t}$, but the revised estimate (which is the fully-revised value in this simple illustration) adds this term: $y_{t}^{t+2}=y_{t}=\alpha y_{t-1}+\eta_{t}+v_{t}$. A characteristic of news is that the revised value is unpredictable from information available at the time of the first estimate, or in other words, the revision $y_{t}^{t+2}-y_{t}^{t+1}=v_{t}$ is not systematically related to $y_{t}^{t+1}$. But the news revision clearly is correlated with the true value. For news, later estimates are more accurate estimates of the true value than earlier estimates (this follows trivially here because $y_{t}^{t+2}=y_{t}$ and so is a perfect estimate). Suppose now that revisions are solely noise, i.e., $\sigma_{\varepsilon}^{2} \neq 0$ (but $\sigma_{v}^{2}=0$ ). For noise, the revised value removes measurement error: the revisions are predictable (based on period $t-1$ information) but are not correlated with the true value.

Consider then a sequence of forecasts made in real time, and in particular, consider the 1-step prediction interval for the period $T$ observation made at time $T$, at which time the available data consists of $\left\{\ldots, \mathbf{y}_{T-2}^{T-1}, \mathbf{y}_{T-1}^{T}\right\}$, where $\mathbf{y}_{T-j-1}^{T-j}=\left[\ldots, y_{T-j-2}^{T-j}, y_{T-j-1}^{T-j}\right]^{\prime}$, for $j=0,1,2, \ldots$ The traditional approach is to specify and estimate the forecasting model using the period $T$-vintage $\left\{\mathbf{y}_{T-1}^{T}\right\}$, on the grounds that this constitutes the best available estimates of $\left\{\ldots, y_{T-2}, y_{T-1}\right\}$, irrespective of whether revisions add news, remove noise, or are some combination of the two. The use of the forecast origin vintage was referred to as end-of-sample (EOS) estimation by Koenig et al. (2003).

$\operatorname{An} \operatorname{AR}(1)$ is estimated on the EOS data:

$$
y_{t}^{T}=\beta y_{t-1}^{T}+e_{t, E O S}, \quad \text { for } t=\ldots, T-2, T-1
$$

and the forecast of $y_{T}$ is given by:

$$
\hat{y}_{T}, E O S=\beta y_{T-1}^{T} \text {. }
$$

As the sample gets large relative to the number of data revisions, it follows that OLS estimation of $\beta$ in (2) will consistently estimate $\alpha$ in (1), because all but the last observation on 
the dependent variable $(t=T-1)$ will equal the true values. This clearly holds more generally for a finite number of data revisions before the truth is revealed, and holds irrespective of whether revisions are news or noise.

Clements and Galvão (2013b) show that if the forecast is conditioned on the vintage- $T$ data (for the $\mathrm{AR}(1)$ model used here this means conditioning on the single observation $y_{T-1}^{T}$, as in (3)) then the optimal value of the parameter $\beta$ in terms of minimizing the expected squared forecast error $\left(E\left[\left(y_{T}^{T+1}-\hat{y}_{T}, E O S\right)^{2}\right]\right)$ is $\beta=\alpha$ for news revisions, but $\beta=\alpha \sigma_{y}^{2}\left(\sigma_{y}^{2}+\sigma_{\varepsilon}^{2}\right)^{-1}$, where $\sigma_{y}^{2}=\operatorname{Var}\left(y_{t}\right)$ and $\sigma_{\varepsilon}^{2}=\operatorname{Var}\left(\varepsilon_{t}\right)$. Hence (3) is the optimal forecast for news, but not for noise. Clements and Galvão (2013b) show more generally that when the true process follows an $\operatorname{AR}(p)$ with $p>1$ then estimation of the model on the vintage- $T$ data (as in the $p>1$ analogue of (2)) will not deliver the optimal parameter vector in a squared error sense for news or noise revisions.

\subsection{News revisions}

As the number of observations gets large, the estimated standard error $\hat{\sigma}_{T-1, E O S}$ from (2) will approach $\sqrt{\sigma_{\eta}^{2}+\sigma_{v}^{2}}$. This is because $E\left(y_{t}^{T}-\beta y_{t-1}^{T}\right)^{2}=E\left(y_{t}-\alpha y_{t-1}\right)^{2}=E\left(\eta_{t}+v_{t}\right)^{2}=\sigma_{\eta}^{2}+$ $\sigma_{v}^{2}$ for $t=\ldots, T-2$, and for $t=T-1, E\left(y_{t}^{T}-\beta y_{t-1}^{T}\right)^{2}=E\left(y_{t}-v_{t}-\alpha y_{t-1}\right)^{2}=E\left(\eta_{t}\right)^{2}=\sigma_{\eta}^{2}$. The effect of the last observation will disappear as $T$ gets large. The Box-Jenkins (BJ) $(1-\gamma)$ level prediction interval is given by:

$$
\left\{\hat{y}_{T, E O S}+z_{\frac{\gamma}{2}} \hat{\sigma}_{T-1, E O S}, \quad \hat{y}_{T, E O S}+z_{1-\frac{\gamma}{2}} \hat{\sigma}_{T-1, E O S}\right\}
$$

where $z_{\gamma}$ is the $\gamma$ quantile of the standard normal, $\gamma=\Phi\left(z_{\gamma}\right)$, and where $\Phi$ denotes the standard normal distribution function.

The expected squared error of the out-of-sample forecast is given by:

$$
\begin{aligned}
E\left(y_{T}^{T+1}-\hat{y}_{T}, E O S\right)^{2} & =E\left(y_{T}-v_{T}-\alpha\left(y_{T-1}-v_{T-1}\right)\right)^{2} \\
& =\sigma_{\eta}^{2}+\alpha^{2} \sigma_{v}^{2}
\end{aligned}
$$

Assuming that the true values follow a stationary $\operatorname{AR}(1),|\alpha|<1$, then the in-sample estimate of uncertainty $\left(\hat{\sigma}_{T-1, E O S}^{2}=\sigma_{\eta}^{2}+\sigma_{v}^{2}\right)$ overstates the true uncertainty surrounding the forecast 
of $y_{T}^{T+1}$. That is, the prediction intervals are too wide. This is the 'true uncertainty' for the model given by (1) when the forecast is conditioned on the vintage- $T$ data, because as noted above EOS delivers the optimal value of the autoregressive parameter in population $(\beta=\alpha)$. The intuitive explanation for the over-estimation of out-of-sample uncertainty is that the insample estimate is based on predicting the revised values, with added news relative to the first estimate, and so is accomplished with less precision than the forecasting of a first estimate $\left(y_{T}^{T+1}\right)$ out-of-sample.

We have assumed that the target is the first estimate rather than the fully-revised value (in our setup, $y_{T}^{T+2}=y_{T}$ ). Real-time forecasting exercises commonly assume that the goal is to forecast a relatively early vintage value, such as the value available one or two quarters after the reference quarter. But for the fully-revised estimate, the BJ interval would now under-estimate the true out-of-sample uncertainty and the actual coverage rate would be less than the nominal:

$$
\begin{aligned}
E\left(y_{T}-\hat{y}_{T}, E O S\right)^{2} & =E\left(y_{T}-\alpha\left(y_{T-1}-v_{T-1}\right)\right)^{2} \\
& =\sigma_{\eta}^{2}+\left(1+\alpha^{2}\right) \sigma_{v}^{2} .
\end{aligned}
$$

\subsection{Noise revisions}

Now suppose revisions reduce noise (and $\sigma_{v}^{2}=0$ ). Consider the in-sample fit of the model. For noise, $E\left(y_{t}^{T}-\beta y_{t-1}^{T}\right)^{2}=E\left(y_{t}-\alpha y_{t-1}\right)^{2}=E\left(\eta_{t}\right)^{2}=\sigma_{\eta}^{2}$ for $t=\ldots, T-2$, and for $t=T-1$, $E\left(y_{t}^{T}-\beta y_{t-1}^{T}\right)^{2}=E\left(y_{t}+\varepsilon_{t}-v_{t}-\alpha y_{t-1}\right)^{2}=E\left(\eta_{t}+\varepsilon_{t}\right)^{2}=\sigma_{\eta}^{2}+\sigma_{\varepsilon}^{2}$, so for large $T$ the insample error variance will be estimated as $\sigma_{\eta}^{2}$.

Consider now the out-of-sample expected squared error:

$$
\begin{aligned}
E\left(y_{T}^{T+1}-\hat{y}_{T}, E O S\right)^{2} & =E\left(y_{T}+\varepsilon_{T}-\alpha\left(y_{T-1}+\varepsilon_{T-1}\right)\right)^{2} \\
& =\sigma_{\eta}^{2}+\left(1+\alpha^{2}\right) \sigma_{\varepsilon}^{2}
\end{aligned}
$$

which exceeds the in-sample error variance. In a reverse of the situation when revisions are news, when revisions reduce noise the in-sample error variance under-estimates the true out-ofsample uncertainty, and consequently the actual coverage of the BJ intervals will fall short of the nominal. Intuitively, when revisions remove noise, the fully-revised data used in the in-sample 
calculation will lead to an under-estimation of the uncertainty that characterizes the out-ofsample estimate. If instead we target the fully-revised value, $E\left(y_{T}-\hat{y}_{T}, E O S\right)^{2}=\sigma_{\eta}^{2}+\alpha^{2} \sigma_{\varepsilon}^{2}$, which still exceeds the in-sample estimate but to a lesser extent.

Note that (5) denotes the out-of-sample uncertainty given by the model estimated by EOS. However, as noted above it does not reflect the minimum level of out-of-sample uncertainty attainable since $\alpha$ does not minimize the expected squared error loss: use of $\phi=\alpha \sigma_{y}^{2}\left(\sigma_{y}^{2}+\sigma_{\varepsilon}^{2}\right)^{-1}$ in place of $\alpha$ in the forecast function would result in a smaller expected squared error. (Simple algebra confirms that $\delta=\phi$ is the value of $\delta$ which minimizes $\left.E\left(y_{T}^{T+1}-\delta y_{T-1}^{T}\right)^{2}\right)$. Hence when there are noise revisions EOS estimation will result in out-of-sample uncertainty being larger than expected given the model's in-sample fit, and larger than is feasible (given the information set consisting of the vintage- $T$ data).

To summarize: prediction intervals will be too wide if data revisions are news (and the aim is to forecast an early vintage, otherwise they will be too narrow), but too narrow if revisions reduce noise.

In a pseudo out-of-sample forecasting exercise, e.g., using the data vintage $\left\{\mathbf{y}_{T-1}^{T+n}\right\}, n \gg 0$, to forecast $y_{T}$, there are no data vintage effects and in-sample and out-of-sample uncertainty would match save for small-sample effects.

\section{A general statistical framework}

Having illustrated the effects of data revisions on the coverage of prediction intervals (and the estimation of forecast uncertainty more generally) in a simple setup, in this section we suppose the true process follows an $\operatorname{AR}(p)$, and allow multiple vintage estimates of each observation. We use the framework in Jacobs and van Norden (2011), as applied by Clements and Galvão (2013b). The period $t+s$ vintage estimate of the value of $y$ in period $t$, denoted $y_{t}^{t+s}$, where $s=1, \ldots, l$, consists of the true value $y_{t}$, as well as news and noise components, $v_{t}^{t+s}$ and $\varepsilon_{t}^{t+s}$, so that $y_{t}^{t+s}=y_{t}+v_{t}^{t+s}+\varepsilon_{t}^{t+s}$. The $l$ vintage estimates of $y_{t}$, namely, $y_{t}^{t+1}, \ldots, y_{t}^{t+l}$ are stacked in the vector $\mathbf{y}_{t}=\left(y_{t}^{t+1}, \ldots, y_{t}^{t+l}\right)^{\prime}$, and similarly $\varepsilon_{t}=\left(\varepsilon_{t}^{t+1}, \ldots, \varepsilon_{t}^{t+l}\right)^{\prime}$ and $\mathbf{v}_{t}=$ $\left(v_{t}^{t+1}, \ldots, v_{t}^{t+l}\right)^{\prime}$, so that:

$$
\mathbf{y}_{t}=\mathbf{i} y_{t}+\mathbf{v}_{t}+\varepsilon_{t}
$$


where $\mathbf{i}$ is a $l$-vector of ones. We suppose $y_{t}$ is an $\operatorname{AR}(p)$ with iid disturbances $R_{1} \eta_{1 t}$, plus a sum of $l$ news components $v_{i, t}$ :

$$
y_{t}=\alpha_{0}+\sum_{i=1}^{p} \alpha_{i} y_{t-i}+R_{1} \eta_{1 t}+\sum_{i=1}^{l} v_{i, t}
$$

where $v_{i, t}=\sigma_{v_{i}} \eta_{2 t, i}($ for $i=1, \ldots, l)$ and both $\eta_{1 t}$ and $\eta_{2 t, i}$ are $i i d(0,1)$. We let $\alpha(L)=\sum_{i=1}^{p} \rho_{i} L^{i}$ and assume that the roots of $(1-\alpha(L))=0$ lie outside the unit circle, so that $y_{t}$ is a stationary process. The news and noise components of each vintage in $\mathbf{y}_{t}$ are:

$$
\mathbf{v}_{t}=\left[\begin{array}{c}
v_{t}^{t+1} \\
v_{t}^{t+2} \\
\vdots \\
v_{t}^{t+l}
\end{array}\right]=-\left[\begin{array}{c}
\sum_{i=1}^{l} v_{i, t} \\
\sum_{i=2}^{l} v_{i, t} \\
\vdots \\
v_{l, t}
\end{array}\right], \quad \varepsilon_{t}=\left[\begin{array}{c}
\varepsilon_{t}^{t+1} \\
\varepsilon_{t}^{t+2} \\
\vdots \\
\varepsilon_{t}^{t+l}
\end{array}\right]=\left[\begin{array}{c}
\sigma_{\varepsilon_{1}} \eta_{3 t, 1} \\
\sigma_{\varepsilon_{2}} \eta_{3 t, 2} \\
\vdots \\
\sigma_{\varepsilon_{l}} \eta_{3 t, l}
\end{array}\right]
$$

where $\eta_{3 t, i}$ is $i i d(0,1)$. The shocks are also mutually independent, that is, if $\boldsymbol{\eta}_{t}=\left[\eta_{1 t}, \boldsymbol{\eta}_{2 t}^{\prime}, \boldsymbol{\eta}_{3 t}^{\prime}\right]$, then $E\left(\boldsymbol{\eta}_{t}\right)=0$, with $E\left(\boldsymbol{\eta}_{t} \boldsymbol{\eta}_{t}^{\prime}\right)=I$.

This generalizes the analysis in section 2 in a straightforward way. Note that $y_{t}^{t+1}=$ $\alpha_{0}+\sum_{i=1}^{p} \alpha_{i} y_{t-i}+R_{1} \eta_{1 t}+\varepsilon_{t}^{t+1}$, whereas later estimates include news. For the $n$th estimate, for example, where $1<n<l$, we have $y_{t}^{t+n}=\alpha_{0}+\sum_{i=1}^{p} \alpha_{i} y_{t-i}+R_{1} \eta_{1 t}+\sigma_{\varepsilon_{n}} \eta_{3 t, n}+\sum_{i=1}^{n-1} v_{i, t}$, which is a more accurate estimate of $y_{t}$ than $y_{t}^{t+1}$, as it includes the news terms $\left(\sum_{i=1}^{n-1} v_{i, t}\right)$ which comprise $y_{t}$. As noted by Mankiw and Shapiro (1986), news revisions imply that $\operatorname{var}\left(y_{t}^{t+1}\right)<$ $\operatorname{var}\left(y_{t}^{t+l}\right)$, while noise revisions imply that $\operatorname{var}\left(y_{t}^{t+1}\right)>\operatorname{var}\left(y_{t}^{t+l}\right)$, assuming that later estimates are less 'noisy' $\left(\sigma_{\varepsilon_{i}}>\sigma_{\varepsilon_{i+1}}\right)$. If $\sigma_{v_{l}}=0$ and $\sigma_{\varepsilon_{l}}=0$ the $l$-vintage value is the true value, $y_{t}^{t+l}=y_{t}$. The assumption that $y_{t}$ is a stationary process ensures that $\mathbf{y}_{t}$ is a stationary process from (6), as both the news and noise terms are stationary. We have assumed for simplicity that both noise and news revisions are zero mean.

The model to be estimated is now given by:

$$
y_{t}^{T}=\beta_{0}+\sum_{i=1}^{p} \beta_{i} y_{t-i}^{T}+e_{t, E O S}, \quad \text { for } t=\ldots, T-2, T-1,
$$

which generalizes (2) to include $p$ autoregressive lags and an intercept. As in section 2 , we make 
the simplifying assumption that EOS is equivalent to estimating the model on fully-revised data. Hence $\beta_{i}=\alpha_{i}$ for $i=0,1, \ldots, p$. Formally this requires that the estimation sample $T$ is large relative to $l$, and that $\sigma_{v_{l}}=\sigma_{\varepsilon_{l}}=0$. This allows us to focus on the first-order effects of data revisions on prediction intervals. Then the population value of the in-sample standard deviation for (9) is equivalent to that from (7), and is given by:

$$
\hat{\sigma}_{T-1, E O S}=\sqrt{E\left[\left(R_{1} \eta_{1 t}+\sum_{i=1}^{l} v_{i, t}\right)^{2}\right]}=\sqrt{R_{1}^{2}+\sum_{i=1}^{l} \sigma_{v_{i}}^{2}} .
$$

The 1-step ahead expected squared forecast error is given by:

$$
\begin{aligned}
E\left[\left(y_{T}^{T+1}-\hat{y}_{T, E O S}\right)^{2}\right] & =E\left[\left(\sum_{i=1}^{p} \alpha_{i}\left(y_{T-i}-y_{T-i}^{T}\right)+R_{1} \eta_{1 T}+\varepsilon_{T}^{T+1}\right)^{2}\right] \\
& =\sum_{i=1}^{p} \alpha_{i}^{2}\left(\sigma_{\varepsilon_{i}}^{2}+\sum_{j=i}^{l} \sigma_{v_{i}}^{2}\right)+R_{1}^{2}+\sigma_{\varepsilon_{1}}^{2}
\end{aligned}
$$

assuming the forecast-origin vintage is dated $T$, and where we have substituted for $y_{T}^{T+1}$ and $\hat{y}_{T, E O S}=\alpha_{0}+\sum_{i=1}^{p} \alpha_{i} y_{T-i}^{T}$, the latter using the true process parameter values, but conditioning the forecast on the $T$-vintage observations. The expression also assumes $l \geqslant p$.

Now consider news revisions. The expression (11) simplifies to $E\left[\left(y_{T}^{T+1}-\hat{y}_{T, E O S}\right)^{2}\right]=$ $\sum_{i=1}^{p} \alpha_{i}^{2} \sum_{j=i}^{l} \sigma_{v_{j}}^{2}+R_{1}^{2}$. Hence out-of-sample uncertainty will be less than in-sample uncertainty $\left(\sigma_{T-1, E O S}^{2}\right.$ defined by $\left.(10)\right)$ when:

$$
\sum_{i=1}^{p} \alpha_{i}^{2} \sum_{j=i}^{l} \sigma_{v_{j}}^{2}<\sum_{i=1}^{l} \sigma_{v_{i}}^{2}
$$

As shown in section 2 this holds when $p=l=1$, given that $\left|\alpha_{1}\right|<1$, but need not hold more generally. For $p>1$, the expected squared error may exceed (the square of) (10) when the $\left|\alpha_{i}\right|$ are large, but generally, one might expect the prediction intervals based on EOS would tend to overstate the uncertainty surrounding future observations when revisions are news.

When revisions are noise, the reverse is true, as then $E\left[\left(y_{T}^{T+1}-\hat{y}_{T, E O S}\right)^{2}\right]=\sum_{i=1}^{p} \alpha_{i}^{2} \sigma_{\varepsilon_{i}}^{2}+$ $R_{1}^{2}+\sigma_{\varepsilon_{1}}^{2}>\sigma_{T-1, E O S}^{2}=R_{1}^{2}$. These findings match those in section 2 : the analysis in that section 
is a special case of the results presented here, with $p=1$ and $l=1$.

As shown by Clements and Galvão (2013b), given that the forecasts condition on $y_{T-1}^{T}, y_{T-2}^{T}, \ldots y_{T-p}^{T}$, the EOS population parameters (which equal those of the true process) are not optimal for news, when $p>1$, or for noise for all $p$. Hence EOS prediction intervals are too narrow for the modelimplied out-of-sample uncertainty when there is noise, and may be too wide or narrow when there is news, although for variables with moderate dependence will be too wide. Moreover, the results of Koenig et al. (2003) and Clements and Galvão (2013b) suggest that EOS forecasts are not optimal in population in a squared-error sense.

A solution to the problem of obtaining correctly-sized prediction intervals in real-time is to use RTV-estimation. This was suggested by Koenig et al. (2003) for first-moment prediction, and further considered by Clements and Galvão (2013b) with an emphasis on autoregressive processes. In the following section we discuss RTV-estimation, and show that it provides intervals with correct coverage.

\section{RTV-estimation}

US NIPA data are typically subject to revision for up to three and a half years after the first estimate is published. Koenig et al. (2003) note that EOS implies that a large part of the data used in model estimation has been revised many times, while the forecast is conditioned on data that has been just released or only revised a few times. That is, the data vector $\mathbf{y}_{T-1}^{T}=\left[\ldots, y_{T-2}^{T}, y_{T-1}^{T}\right]^{\prime}$ comprises the first estimate of $y_{T-1}$, the first revision (i.e., the second estimate) of $y_{T-2}$, and so on up to mature data for the earlier data periods. They show that more accurate forecasts can be achieved (in principle) by not mixing mature and lightly-revised data, and instead advocate using 'real-time vintage' (RTV). The forecasting model is estimated on data of a similar maturity to the data on which the forecast is conditioned. This is Strategy 1 of Koenig et al. (2003), p.620, which we refer to throughout as RTV-estimation, or the use of RTV data.

We maintain the assumption made throughout that forecasts will be conditioned on data estimates from the latest-available vintage at the time the forecasts are made. For an $\operatorname{AR}(p)$, this means that the forecast will be conditioned on $\mathbf{y}_{T-1}^{T}=\left[y_{T-1}^{T}, y_{T-2}^{T}, \ldots y_{T-p}^{T}\right]^{\prime}$. The RTV 
approach estimates the $\operatorname{AR}(p)$ on matching early-release data:

$$
y_{t-1}^{t}=\beta_{0}+\sum_{i=1}^{p} \beta_{i} y_{t-1-i}^{t-1}+e_{t, R T V}, \quad \text { for } t=\ldots, T-1, T \text {, }
$$

and the forecast of $y_{T}$ is $\hat{y}_{T}, R T V=\beta_{0}+\beta_{1} y_{T-1}^{T}+\ldots+\beta_{p} y_{T-p}^{T}$. For notational simplicity, let $\boldsymbol{\beta}=\left[\beta_{1} \ldots \beta_{p}\right]^{\prime}$, so that we can equivalently write $\hat{y}_{T, R T V}=\beta_{0}+\beta^{\prime} \mathbf{y}_{T-1}^{T}$. Clements and Galvão (2013b) show that for a general model of data revisions, as in Jacobs and van Norden (2011), the solution $\left(\phi_{0}^{*}, \phi^{*}\right)$ of:

$$
\underset{\phi_{0}, \boldsymbol{\phi}}{\arg \min } E\left[\left(y_{T}^{T+1}-\phi_{0}-\boldsymbol{\phi}^{\prime} \mathbf{y}_{T-1}^{T}\right)^{2}\right]
$$

is satisfied by the RTV-population values: $\beta_{0}=\phi_{0}^{*}$, and $\boldsymbol{\beta}=\boldsymbol{\phi}^{*}$. That is, given that the forecast is conditioned on $\mathbf{y}_{T-1}^{T}$, RTV will deliver (in population) the values of the intercept and autoregressive parameters which minimize the expected squared error. Clements and Galvão (2013b) show that $\left(\phi_{0}^{*}, \phi^{*}\right)$ depend on the nature of data revisions (news or noise, whether they are zero mean, etc.).

It follows immediately that RTV-estimation will provide correct assessments of out-ofsample uncertainty. The population values of the OLS estimators of the unknown parameters in (12) are the same as the solution to:

$$
\left(\beta_{0}^{*}, \boldsymbol{\beta}^{*}\right)=\underset{\beta_{0}, \boldsymbol{\beta}}{\arg \min } E\left[\left(y_{t}^{t+1}-\beta_{0}-\boldsymbol{\beta}^{\prime} \mathbf{y}_{t-1}^{t}\right)^{2}\right]
$$

A typical observation on the LHS and RHS variables in (12) is $\left\{y_{t}^{t+1}, \mathbf{y}_{t-1}^{t}=\left(y_{t-1}^{t} \ldots y_{t-p}^{t}\right)^{\prime}\right\}$, which is a covariance stationary process.

The estimation loss function (14) is identical to the real-time forecast loss function (13). Clements and Galvão (2013b) stress that the solutions to the two in terms of $\left(\phi_{0}, \boldsymbol{\phi}\right)$ and $\left(\beta_{0}, \boldsymbol{\beta}\right)$ coincide, $\beta_{0}^{*}=\phi_{0}^{*}$ and $\boldsymbol{\beta}^{*}=\phi^{*}$, and thus RTV-estimation delivers optimal forecasts. For our purposes, note that the values of the functions in (13) and (14) evaluated at the RTV population parameters are identical, implying in addition that the in-sample estimate of uncertainty from RTV-estimation will provide a reliable guide to out-of-sample forecasting. This holds for general revisions processes, such as that considered by Jacobs and van Norden (2011), provided the true 
process and the data revisions are stationary.

An alternative to using RTV data is to use models that draw on the multiple estimates of each observation which are typically available. There are many such models, e.g., Harvey, McKenzie, Blake and Desai (1983), Howrey (1984), Patterson (1995, 2003), Jacobs and van Norden (2011), Cunningham et al. (2009) and Garratt et al. (2009, 2008). In terms of firstmoment prediction, Clements and Galvão (2013b) compare the accuracy of RTV with forecasts from a vector autoregression (VAR), that models the relationships between the multiple-vintage estimates (in the spirit of recent work by Garratt et al. $(2008,2009)$ ), and with the approach of Kishor and Koenig (2012), which specifies a model for the data revisions process which is estimated along side a VAR for the 'post-revision' data. They find the performance of these more elaborate models is on a par with RTV-estimation of the AR (for first-moment prediction). In this paper we do not examine the potential usefulness of these multiple-vintage models for calculating prediction intervals.

To end this section, we illustrate the general results using the simple example of section 2 , that is, a zero-mean $\mathrm{AR}(1)$ with a single revision. In section 2 this example was used to motivate the problems with the standard approach. Here we show that RTV provides correctlysized prediction intervals in this setup.

\subsection{News revisions}

The population value of $\beta$ in the RTV-regression model:

$$
y_{t-1}^{t}=\beta y_{t-2}^{t-1}+e_{t}
$$

is $\beta=\operatorname{Cov}\left(y_{t-1}^{t}, y_{t-2}^{t-1}\right) / \operatorname{Var}\left(y_{t-2}^{t-1}\right)=\alpha$. This comes from:

$$
\begin{aligned}
\operatorname{Cov}\left(y_{t-1}^{t}, y_{t-2}^{t-1}\right) & =E\left[\left(\alpha y_{t-2}+\eta_{t-1}\right)\left(y_{t-2}-v_{t-2}\right)\right]=\alpha \sigma_{y}^{2}-\alpha \sigma_{v}^{2}, \\
\operatorname{Var}\left(y_{t-2}^{t-1}\right) & =\operatorname{Var}\left(y_{t-2}-v_{t-2}\right)=\sigma_{y}^{2}+\sigma_{v}^{2}-2 \operatorname{Cov}\left(y_{t-2}, v_{t-2}\right)=\sigma_{y}^{2}-\sigma_{v}^{2}
\end{aligned}
$$

This result is a special case of the general formulae presented in Clements and Galvão (2013b). Further, the RTV parameter $\beta$ only equals the autoregressive parameter of the true process $(\alpha)$ for the AR(1) with news, as here. Then the in-sample error variance is based on $\operatorname{Var}\left(y_{t-1}^{t}-\alpha y_{t-2}^{t-1}\right)$ 
which on substituting for $y_{t-1}^{t}$ and $y_{t-2}^{t-1}$ is equal to $\sigma_{\eta}^{2}+\alpha^{2} \sigma_{v}^{2}$. It is a simple matter to show that this equals the out-of-sample uncertainty: $E\left[\left(y_{T}^{T+1}-\hat{y}_{T}, R T V\right)^{2}\right]=E\left[\left(y_{T}-v_{T}-\alpha\left(y_{T-1}-v_{T-1}\right)\right)^{2}\right]=$ $\sigma_{\eta}^{2}+\alpha^{2} \sigma_{v}^{2}$.

\subsection{Noise revisions}

When there are noise revisions, it follows that in population:

$$
\beta=\phi^{*}=\alpha \frac{\sigma_{y}^{2}}{\sigma_{y}^{2}+\sigma_{\varepsilon}^{2}} .
$$

The in-sample error variance and the out-of-sample squared forecast error are equal, and prediction intervals based on the former will have correct conditional coverage. The in-sample error variance is:

$$
\operatorname{Var}\left(y_{t-1}^{t}-\beta y_{t-2}^{t-1}\right)=\operatorname{Var}\left[y_{t-1}+\varepsilon_{t-1}-\beta\left(y_{t-2}+\varepsilon_{t-2}\right)\right]
$$

and the out-of-sample squared forecast error is:

$$
\operatorname{Var}\left(y_{T}^{T+1}-\hat{y}_{T}, R T V\right)^{2}=\operatorname{Var}\left[y_{T}+\varepsilon_{T}-\beta\left(y_{T-1}+\varepsilon_{T-1}\right)\right] .
$$

The two variances are equal given the assumed stationarity of $\left\{y_{t}\right\}$ and $\left\{\varepsilon_{t}\right\}$.

Note that for both news and noise revisions, the equality of the in and out-of-sample error variances rests on forecasting the first estimate of $y_{T}$.

\section{$5 \quad$ Predicting revised data}

So far we have focused on predicting the first data release, and have shown that RTV as implemented in section 4 is a simple and viable method of obtaining correctly-sized prediction intervals. In the motivating discussion of the shortcomings of EOS in section 2 we showed that EOS intervals would also be incorrectly-sized for assessing the uncertainty around the fullyrevised data. In this section we establish that EOS intervals are invalid in the general setup of section 3, where we allow an $\operatorname{AR}(p)$ and multiple revisions. For forecasting fully-revised data, i.e., $y_{T}^{T+l}$, where $y_{T}^{T+l}=y_{T}$, the expression for the 1-step ahead expected squared forecast error 
(corresponding to (11)) is given by:

$$
\begin{aligned}
E\left[\left(y_{T}-\hat{y}_{T, E O S}\right)^{2}\right] & =E\left[\left(\sum_{i=1}^{p} \alpha_{i}\left(y_{T-i}-y_{T-i}^{T}\right)+R_{1} \eta_{1 T}-v_{T}^{T+1}\right)^{2}\right] \\
& =\sum_{i=1}^{p} \alpha_{i}^{2}\left(\sigma_{\varepsilon_{i}}^{2}+\sum_{j=i}^{l} \sigma_{v_{i}}^{2}\right)+R_{1}^{2}+\sum_{i=1}^{l} \sigma_{v_{i}}^{2}
\end{aligned}
$$

which exceeds the in-sample uncertainty (the square of (10)) by the first term. Hence whether revisions are news or noise, the standard approach to estimating out-of-sample uncertainty about the revised value will tend to underestimate that uncertainty, resulting in prediction intervals which are too narrow. This differs from the results obtained for forecasting the first release, when intervals were too wide for news but too narrow for noise.

Equation (16) also suggests the EOS intervals might be expected to be relatively more accurate for revised data than first-release data, because the first term in this expression will be small when the $\left|\alpha_{i}\right|$ are small (e.g., consider a variable modelled by an $\operatorname{AR}(1)$ with $\alpha_{1}=0.3$ ). This contrasts the findings for predicting first-release values in section 3 , where the difference between the EOS in-sample and out-of-sample uncertainty estimates do not vanish as the $\left|\alpha_{i}\right|$ get small. (For example, compare (10) with (11)).

Intuitively, when EOS is used and the goal is to predict revised data, the only distortion (between the model's in-sample estimate of uncertainty and out-of-sample uncertainty) is from the forecast being conditioned on early-vintage observations: were the forecasts conditioned on the (unknown at the time of forecasting) fully-revised values of the observations, the intervals would have the correct coverage in population. This contrasts with forecasting the first-release value, where conditioning on fully-revised data would not deliver correctly-sized intervals.

The logic of the RTV approach in section 4 suggests a simple modification for forecasting revised data. Suppose we wish to forecast $y_{T}^{T+n}$ (where $n \leq l$, and $n=l$ corresponds to forecasting the fully-revised data), then (12) is adapted to:

$$
y_{t-1}^{t+n-1}=\beta_{0}+\sum_{i=1}^{p} \beta_{i} y_{t-1-i}^{t-1}+e_{t, R T V} .
$$

That is, the dependent variable $y_{t-1}^{t}$ is replaced $y_{t-1}^{t+n-1}$. When the forecast origin data vintage is 
$T$, the estimation sample is of necessity reduced from $t=\ldots, T-1, T$ to $t=\ldots, T-n, T-n+1$, with the loss of the last $n-1$ observations, relative to when the goal is to forecast the first release.

It follows immediately that the adapted RTV-estimation will provide correct assessments of out-of-sample uncertainty. The population values of the OLS estimators of the unknown parameters in (17) are the same as the solution to:

$$
\left(\beta_{0}^{*}, \boldsymbol{\beta}^{*}\right)=\underset{\beta_{0}, \boldsymbol{\beta}}{\arg \min } E\left[\left(y_{t}^{t+n}-\beta_{0}-\boldsymbol{\beta}^{\prime} \mathbf{y}_{t-1}^{t}\right)^{2}\right]
$$

Writing the forecast as $\hat{y}_{T}, R T V=\beta_{0}^{*}+\boldsymbol{\beta}^{* \prime} \mathbf{y}_{T-1}^{T}$, the expected squared-error out-of-sample is given by:

$$
E\left[\left(y_{T}^{T+n}-\beta_{0}^{*}-\boldsymbol{\beta}^{* \prime} \mathbf{y}_{T-1}^{T}\right)^{2}\right]
$$

Then the expected squared error out-of-sample (19) matches the in-sample error variance (evaluated at the adapted RTV population values), and prediction intervals based on the adapted RTV method are correctly-sized for the $n$th data vintage value of $y_{T}$. Because the in-sample estimation criterion matches the out-of-sample loss function, the resulting forecasts are optimal for $y_{T}^{T+n}$ given that the forecasts are conditioned on $\mathbf{y}_{T-1}^{T}$.

Although not explicitly shown, it also follows that the RTV method described in section 4 for predicting the first release would not generate correctly-sized intervals for forecasting revised data irrespective of whether revisions are news or noise.

In practice setting $n=14$, say, to include the 3 rounds of annual revisions to which US Bureau of Economic Analysis data are subject might have a negative impact in terms of parameter estimation uncertainty from the loss of data. A pragmatic response is to set $n=3$ when we consider forecasting revised data (as opposed to $n=1$ for the first release) as the third estimates are based on reasonably complete data relative to the first release. 


\section{BJ prediction intervals for AR models estimated using RTV and EOS}

We consider 25 US macro variables which are subject to data revisions. The variables are described in table 1. The data vintages are taken from the Real-Time Data Set for Macroeconomists (RTDSM) of Croushore and Stark (2001). Our first 'vintage-origin' is 1996:Q2, and the last is 2011:Q1, so that we have 15 years of quarterly forecast origins. However, the 1999:Q4 and 2009:Q3 vintages contain missing values for many series and these forecast origins are excluded. In order to estimate the models by EOS, we require that each data vintage provides a long enough history of past observations. We use a rolling-window forecasting scheme, where for the first vintage origin of 1996:Q2, we use data from 1984 onwards. However, for RTV, we need data vintages going back to 1984 to have data over the same historical period. That is, we require an additional 12 years of data-vintages for RTV estimation. This requirement was satisfied for the 25 variables forecast in this study.

For all but one of the variables (ruc - the unemployment rate) we model, and evaluate forecasts of, the first difference of the natural logarithm. The variable ruc is modelled and forecast untransformed.

To focus on the RTV versus EOS issue, in all cases we use $\operatorname{AR}(2)$ models (i.e., two autoregressive lags), although the number of lags could be selected for each variable at each forecast origin using an information criterion such as BIC. The real-time (EOS) forecasting performance of the AR models (estimated by EOS) has been shown to be improved by discarding the preGreat Moderation data (Clements (2015)), which is the reason we set the initial start point of the estimation period to 1984. In the first instance we use first-release actual values. The analysis in section 5 suggests that the distortionary effects of EOS estimation will be relatively muted for forecasting revised data, so we begin with forecasting first-release values where we expect the problems with EOS to be clearest. So the actual values are taken from the vintage available one quarter after the target quarter. To evaluate the forecast of 1996:Q2, from the first vintage origin of 1996:Q2, for example, we take the observation from the 1996:Q3 data vintage.

Table 2 records our first set of results: the relative magnitudes of the models' estimated 
standard errors for RTV and EOS estimation; $t$-statistics of the null that revisions are news; $t$-statistics of the null that revisions are noise; and the actual coverage rates of one-step ahead BJ intervals. The tests for news and noise are based on the revisions between the first-estimates $y_{t}^{t+1}$, and the data available some three and a half years later $y_{t}^{t+15}$, which includes the three rounds of annual regular revisions. We test for news and noise revisions using, respectively:

$$
y_{t}^{t+1}-y_{t}^{t+15}=\alpha+\beta_{n e} y_{t}^{t+1}+\omega_{t}
$$

and:

$$
y_{t}^{t+1}-y_{t}^{t+15}=\alpha+\beta_{n o} y_{t}^{t+15}+\omega_{t} .
$$

We find that 9 of the 25 variables have data revisions which are news, in that we do not reject $\beta_{n e}=0$, but we do reject $\beta_{n o}=0$, at conventional levels. For all of these variables the in-sample standard deviation estimated by EOS exceeds the RTV estimate. For the 7 variables for which we do not reject $\beta_{n o}=0$ but do reject $\beta_{n e}=0$, implying noise revisions, we find the reverse - the EOS standard deviation is smaller than the RTV estimate. Hence for the 16 variables which can be categorized as news or noise, the relative magnitudes of the in-sample standard deviations are as expected given the analysis in section 4. The remaining variables cannot be characterized as purely news or noise, so it is not clear what one would expect to find. Aruoba (2008) and Corradi, Fernandez and Swanson (2009) provide recent extensions to testing for the properties of data revisions, but for our purpose of providing a broad classification of each series as being subject to news or noise revisions the standard approach suffices.

In terms of out-of-sample performance, for around $80 \%$ of the variables the RTV intervals are more accurate than the EOS intervals, in the sense that the actual coverage rates are closer to the nominal rates. The actual coverage rates are shown for each variable for the RTV and EOS intervals in table 2 , but summarizing across all variables, we find that the coverage rates of RTV intervals are closer to the nominal for 20, 21 and 18 variables for the $50 \%, 75 \%$ and $90 \%$ nominal intervals, respectively. Furthermore, our analysis suggests EOS-interval coverage should exceed that of RTV intervals for variables with news revisions, with the opposite holding for noise revisions. Of the 9 variables categorized as having news revisions, EOS-interval coverage is greater for either all, or all but one, of these variables (depending on the nominal interval size). 
Of the 7 variables with noise revisions, the RTV coverage rate is greater for all 7 variables for the $50 \%$ and $75 \%$ intervals (and for all but one for the $90 \%$ intervals).

As a robustness check, we calculated the statistics reported in table 2 for models with an autoregressive order of one, and found the results were qualitatively unchanged (not reported to save space).

We then supposed the goal of the analysis was to forecast the revised data, either the third estimate, or the fully-revised data (taken to be the value in the 2012:Q3 vintage). We compared the accuracy of intervals based on adapted RTV estimation (with $n=3$ in (17), so optimal in population for the third estimate) against intervals based on EOS estimation, for the 25 variables in our study, and using the same setup as for forecasting the first-release data. We found that the adapted RTV interval coverage rates were closer to the nominal rates than the EOS intervals for 18, 22 and 16 variables, for the 50\%, $75 \%$ and $90 \%$ coverage-rate intervals, respectively. Hence RTV based on (17) works reasonably well in terms of estimating the uncertainty surrounding the third estimates. For forecasting the fully-revised there was little to choose between RTV (with $n=3$ ) and EOS, with the RTV intervals now being more accurate for 14, 12 and 11 of the variables. In principle $n$ should be set to include the three rounds of annual revisions embodied in the final data (so $n=14$, say), but this would have meant omitting the last 3 and a half years of quarterly data from the estimation sample at each forecast origin.

RTV estimation is expected to generate more accurate BJ-intervals than EOS because the RTV-estimate of the in-sample standard deviation more accurately reflects the out-of-sample uncertainty. Table 2 is designed to show the relationship between the in-sample standard deviations, and the average interval coverage rates. However, obtaining the correct coverage 'on average' is a minimal requirement of a sequence of prediction intervals. To see this, let $L_{t \mid t-1}(p)$ and $U_{t \mid t-1}(p)$ denote the lower and upper limits of a 1-step ahead prediction interval with nominal coverage $p$, and let $I_{t}=1$ denote a 'hit', defined as:

$$
I_{t}= \begin{cases}1 & \text { if } y_{t} \in\left(L_{t \mid t-1}(p), U_{t \mid t-1}(p)\right), \\ 0 & \text { otherwise }\end{cases}
$$

for a sequence of forecasts $\left(\left\{L_{t \mid t-1}(p), U_{t \mid t-1}(p)\right\}\right)$ and realizations $\left(\left\{y_{t}\right\}\right), t=1,2, \ldots, N$. Cor- 
rect unconditional coverage holds when $E\left(I_{t}\right)=p$, assessed by whether the sample mean $\frac{1}{N} \sum_{t=1}^{N} I_{t}$ is close to $p$. A more stringent criterion is that the occurrences of 1's and 0's are unpredictable - for a given information set $\mathcal{I}_{t}$ (where $\mathcal{I}_{t}=\left\{I_{t}, I_{t-1}, \ldots\right\}$ at a minimum) we require $E\left(I_{t} \mid \mathcal{I}_{t-1}\right)=p$. When $\mathcal{I}_{t}=\left\{I_{t}, I_{t-1}, \ldots\right\}$, this is equivalent to saying that $\left\{I_{t}\right\}$ is iid Bernoulli with parameter $p$. This is a joint test, and Christoffersen (1998) presents simple likelihoodbased tests of the component parts: correct unconditional coverage $\left(E\left(I_{t}\right)=p\right)$; independence (against a first-order Markov chain structure for $\left\{I_{t}\right\}$ ); as well as of the joint hypothesis of correct conditional coverage. The test for independence will have power to detect (unmodelled) changes in the volatility (because hits will tend to be clustered during the relatively low volatility periods) as well as dynamic mis-specification of the model generating the forecasts. For example, prediction intervals generated by an $\mathrm{AR}(1)$ (say), when the data generating process is an $\operatorname{AR}(2)$ with gaussian disturbances, will have correct unconditional coverage but not correct conditional coverage. Corradi and Swanson (2006) provide a related discussion in the context of density forecasting.

Table 3 reports the $p$-values for the tests of correct unconditional coverage (UC), independence (IND) and conditional coverage (CC) for each variable, for intervals of three nominal sizes $(50 \%, 75 \%$ and $90 \%)$. The table assumes the goal is to predict the first-release values. The penultimate row of the table reports the number of variables for which the null of the corresponding test is rejected (when the test is conducted at the $5 \%$ level). The EOS intervals are rejected for more variables than the RTV intervals, and the rejections are mainly of correct unconditional coverage (or 'bias'), rather than of the test for independence. This indicates that many of the differences between the nominal and actual coverage rates recorded in table 2 for the EOS intervals are statistically significant. When we used adapted-RTV to predict the third-estimates, there was little to choose between RTV and EOS in terms of rejection rates across the set of 25 variables (see last row of table).

Interval coverage rates may also differ because the interval is located about an inaccurate point forecast, and not just because of the scale of the predictive distribution underlying the interval. However, not withstanding the superiority of RTV-estimation in principle for firstmoment prediction (see Koenig et al. (2003) and Clements and Galvão (2013b)), for our setup we find little to choose between the two for the majority of variables, when the point forecasts 
of the first-release values are evaluated by RMSE: see table 4 .

Table 4 shows that RTV improves accuracy by $5 \%$ on RMSE for forecasting output growth. But with one or two exceptions, this is at the top end of the gains to RTV, and a number of the entries exceed one, suggesting EOS is more accurate for those variables. This suggests that the RTV-intervals chiefly benefit from more accurate estimates of scale rather than location. The general point is that RTV estimation in practical forecasting may matter more for secondmoment type forecasts (such as prediction intervals) than for point forecasting.

This last point is supported by the results in table 4 for bivariate ADL (autoregressivedistributed lag) models, where again the aim is to predict the first release. For each of the 25 variables we generate EOS and RTV point forecasts for the 24 possible bivariate ADL models, where we include 2 lags of the dependent variable, and of the explanatory variable. This allow us to assess the relative point forecasting performance of RTV compared to EOS for models with explanatory variables which are subject to revision. The results for the ADL models are no more favourable for RTV than the results for AR models. We present the median, mean, minimum and maximum of the ratios across the 24 ADL models for each variable. There is an ADL model (i.e., an explanatory variable) for which RTV is $10 \%$ more accurate than EOS for forecasting output growth. But equally there is a variable for which EOS is $9 \%$ more accurate for forecasting output growth, and this pattern holds across the majority of variables.

In summary, the empirical estimates of coverage rates are generally in line with the analysis in section 4 , and the value of RTV is largely attributable to more accurate estimates of the scale of the predictive distribution, rather than the location (that is, than of the point prediction).

\section{Simulation study}

We established in section 4 that BJ intervals based on RTV-estimation would be correctly-sized in the presence of data revisions, and that intervals based on EOS-estimation of the model would likely have a coverage in excess of the nominal when data revisions are news, but less than the nominal when data revisions are noise. These statements hold 'in population', that is, when we ignore parameter estimation uncertainty, and when the aim is to predict the firstrelease values. Even in the absence of data revisions, it is well known that BJ-intervals can 
be adversely affected by parameter estimation uncertainty when the sample size is small. In addition, there will be uncertainty about the model order. Having to select appropriate model orders may affect the relative merits of RTV and EOS. Model selection issues are especially pertinent in our context because the optimal model order for RTV-estimation may differ from that using EOS-estimation. To illustrate with a simple case, consider the example in section 4, where the true values follow a zero-mean $\operatorname{AR}(1)$ and there is a single noise revision. Hence the data generating process is given by:

$$
y_{t}=\alpha y_{t-1}+\eta_{t}
$$

and the estimates of $y_{t}$ are given by:

$$
\begin{aligned}
& y_{t}^{t+1}=y_{t}+\varepsilon_{t} \\
& y_{t}^{t+n}=y_{t}
\end{aligned}
$$

for $n=2,3, \ldots$. Let the population first-order RTV-regression be:

$$
y_{t}^{t+1}=\beta y_{t-1}^{t}+e_{t, R T V} .
$$

where from (15) we know that $\beta \neq \alpha$ when $E\left(\varepsilon_{t}^{2}\right) \neq 0$ (i.e., $\beta \neq \alpha$ when there are noise revisions). Substituting for $y_{t}^{t+1}$ and $y_{t-1}^{t}$ from (22) into (23) yields $e_{t, R T V}=y_{t}-\beta y_{t-1}+$ $\varepsilon_{t}-\beta \varepsilon_{t-1}$. Then it follows immediately that the first-order model is dynamically-mis-specified because the second 'lag' $y_{t-2}^{t}$ (here equal to $y_{t-2}$ ) is correlated with the error term $e_{t, R T V}$ :

$$
\operatorname{Cov}\left(e_{t, R T V}, y_{t-2}\right)=\operatorname{Cov}\left(y_{t}-\beta y_{t-1}, y_{t-2}\right)+\operatorname{Cov}\left(\varepsilon_{t}-\beta \varepsilon_{t-1}, y_{t-2}\right)
$$

The second covariance on the RHS is zero, and the first is only zero when $\beta=\alpha$, in which case $y_{t}-\beta y_{t-1}=\eta_{t}$.

For these reasons we investigate the small-sample properties of the procedures by simulation, allowing that the appropriate model orders need to be selected by an information criterion, such as BIC. Of interest is whether RTV-estimation provides accurate intervals in these circumstances. 
Simulating data with revisions requires a complete specification of the data revisions process. This requires giving values to a relatively large number of parameters, and the concomitant concern that the results of the simulation study may be specific to the range of values considered. In order to obtain sensible values for the data generating process, we use the model estimated by Jacobs and van Norden (2011) for US real output growth as our base case. They allow for data revisions to be news or noise, with and without 'spillovers'. We also experiment with a variant in which the standard deviation of the underlying shock is divided by 4 , but the standard deviations of the news and noise disturbances (and all the other parameters) are left unaltered, to gauge the impact of data revisions being more prominent (than they are for real output, as estimated by Jacobs and van Norden (2011)). To save space, we do not repeat the details of their model, except to note that there are four vintage estimates of each time period, and the final is not assumed to reveal the truth (i.e., $y_{t}^{t+4} \neq y_{t}$ ). The estimated parameter values that we use are taken from their Table 1 (p.107). We simulate 25, 000 replications of length $T+1$, after discarding initial observations to remove any dependence on initial conditions, and on each sample we estimate the AR model by EOS and RTV on the first $T$ observations, and calculate a BJ prediction interval for the first estimate of the $T+1$ observation (i.e., $y_{T+1}^{T+2}$ ).

The results are recorded in table 5. For the Jacobs and van Norden (2011) data generating process (top half of table) the RTV intervals are under-sized at small $T$ but approximately correctly-sized at larger $T$. The coverage rates are similar (for a given $T$ ) whether revisions are news of noise, and irrespective of whether there are spillovers: RTV-intervals are largely immune to the effects of data revisions. This is underlined by comparing these estimated coverage rates with those when there are no revisions (recorded at the foot of the table): the two sets are virtually identical. It is well known in the literature that neglecting the uncertainty inherent in the estimated model parameters will lead to under-sized intervals, and this effect is obviously more pronounced at small $T$. By contrast, EOS-estimation is not a reliable method of generating prediction intervals, giving rise to intervals which are under-sized or over-sized, depending on $T$, when revisions are news. When revisions are more prominent (second half of table), the performance of the EOS intervals worsens, and the EOS intervals are clearly under-sized when there is noise. Whereas the performance of the RTV intervals is virtually unaffected. There is little appreciable effect from allowing spillovers. 
Table 6 presents Monte Carlo estimates of coverage rates for forecasting the revised data (here, the fourth estimate). We report results for experiments matching the second half of table 5 (i.e., the more prominent revisions), as well as when the autoregressive parameters are larger (at 0.90 and 0.05 ), in the second half of the table. The table gives the results of using RTV, EOS and adapted RTV. As expected, the costs to using EOS in terms of biased coverage rates are reduced relative to forecasting early-release data. In the top half of the table, for example, the large-sample coverage rates are correct, as are those of adapted RTV (as expected). The intervals for RTV tuned to first-release forecasting are not correct, even in large samples. The small-sample performance of the adapted RTV intervals is adversely affected by the loss of observations. The second half of the table indicates that when the process is highly persistent the EOS intervals may be incorrectly-sized even in large samples, whereas the adapted RTV estimates remain correctly-sized .

Table 7 records the rejection frequencies of the tests for unconditional coverage, independence and conditional coverages, for forecasting the first-release data. Although we include the test for independence (and correct conditional coverage), in the absence of unmodelled timevarying heteroscedasticity we would not expect this test to deviate much from its nominal size, and that transpires to be the case. The rejection frequencies are based on generated sequences of 100 prediction intervals and actuals. The initial sample size is recorded in the table, and the model is re-estimated (by EOS or RTV) on an expanding window of data prior to calculating the interval. For the resulting vector of hits and misses, we calculate the three tests. The table records the rejection frequencies across 10,000 replications of this procedure. For the RTV intervals, there are some minor size distortions at the smaller sample sizes, but otherwise the tests are correctly sized, while the EOS intervals are clearly inadequate, and this is flagged by the tests of coverage. For the selected Monte Carlo data-generation process parameter values, the rejections are greater for noise revisions, and for the $75 \%$ and $90 \%$ nominal intervals are in excess of $50 \%$. We conclude that prediction intervals from RTV-estimation with BIC modelorder specification generates intervals with desirable properties, whilst the traditional approach (EOS-estimation) does not. 


\section{Conclusions}

We have shown that assessments of future macroeconomic uncertainty based on the in-sample fit of a model are likely to be misleading when the variable being modelled is subject to revision. This is particularly true when the aim is to predict an early-vintage estimate of a future observation. Then the data on which the model is estimated will be for the most part fully-revised or mature data, whereas the out-of-sample value is a first-release or only lightly-revised data point. In the context of first-moment prediction Kishor and Koenig (2012) referred to estimating the model on fully-revised data, and conditioning the forecast on only lightly-revised data, as mixing 'apples and oranges'. In the context of second-moment prediction the mismatch results instead from supposing the goodness of fit of the model on the fully-revised data (i.e., the in-sample period) is an accurate representation of the out-of-sample fit.

For forecasting revised or mature values of future observations we show that these concerns are alleviated.

We have shown that a simple solution is to use real-time-vintage (RTV) data. This was proposed by Koenig et al. (2003) in the context of first-moment prediction. Based on the evidence for the 25 macro variables we consider in this paper, RTV-estimation is more beneficial for second-moment forecasting. Its validity in population (abstracting from parameter estimation uncertainty) is easily established. Its good forecast performance has been demonstrated, and is supported by a simulation study.

We have considered autoregressive models in this paper, but the logic of the arguments suggests that the findings carry over immediately to models with explanatory variables, so the problems with using EOS, and the advantages of RTV, are potentially more generally applicable. For example, there has been much interest in measuring macro-uncertainty in the recent literature, driven in large part by the belief that time-varying uncertainty may play an important role in business cycle fluctuations (see, e.g., Bachmann, Elstner and Sims (2013)). Some of the approaches to measuring macro-uncertainty use 'data-rich' modelling environments, and are pseudo real-time, perhaps because of the difficulties of collecting and managing different data vintages at each point in time. Two recent closely-related contributions are Jurado, Ludvigson and Ng (2015) and Henzel and Rengel (2013). As pseudo-real time exercises, the effects of data revisions are side-stepped. However, the measures of macro uncertainty are derived 
from the individual variables' forecast errors, rather than the in-sample fits of the models, and so would be immune to the distorting effects described in this paper if similar exercises were carried out in real time. For example, Jurado et al. (2015) calculate forecast errors for each of a large number of variables using a factor model, and then fit a stochastic volatility model to the forecast errors to obtain individual-variable volatility forecasts, which are then aggregated. Other approaches which use instead the in-sample fit of the model - as in the standard approach to calculating prediction intervals illustrated in this paper - are likely to be misleading in the presence of data revisions. 


\section{References}

Aruoba, S. B. (2008). Data revisions are not well-behaved. Journal of Money, Credit and Banking, 40, 319-340.

Bachmann, R., Elstner, S., and Sims, E. R. (2013). Uncertainty and economic activity: Evidence from Business Survey Data. American Economic Journal: Macroeconomics, 5(2), 217249.

Box, G. E. P., and Jenkins, G. M. (1970). Time Series Analysis, Forecasting and Control. San Francisco: Holden-Day.

Christoffersen, P. F. (1998). Evaluating interval forecasts. International Economic Review, 39, $841-862$.

Clements, M. P. (2015). Real-time factor model forecasting and the effects of instability. Computational Statistics and Data Analysis. doi:10.1016/j.csda.2015.01.011, Forthcoming.

Clements, M. P., and Galvão, A. B. (2012). Improving real-time estimates of output gaps and inflation trends with multiple-vintage VAR models. Journal of Business and $\mathcal{G}$ Economic Statistics, 30(4), 554-562. DOI: 10.1080/07350015.2012/707588.

Clements, M. P., and Galvão, A. B. (2013a). Forecasting with vector autoregressive models of data vintages: US output growth and inflation. International Journal of Forecasting, 29(4), 698 - 714. DOI: 10.1016/j.ijforecast.2011.09.003.

Clements, M. P., and Galvão, A. B. (2013b). Real-time forecasting of inflation and output growth with autoregressive models in the presence of data revisions. Journal of Applied Econometrics, 28(3), 458-477. DOI: 10.1002/jae.2274.

Corradi, V., Fernandez, A., and Swanson, N. R. (2009). Information in the revision process of real-time datasets. Journal of Business and Economic Statistics, 27, 455-467.

Corradi, V., and Swanson, N. R. (2006). Bootstrap conditional distribution tests in the presence of dynamic misspecification. Journal of Econometrics, 133(2), 779-806.

Croushore, D. (2011a). Forecasting with real-time data vintages, chapter 9. In Clements, M. P., and Hendry, D. F. (eds.), The Oxford Handbook of Economic Forecasting, pp. 247-267: Oxford University Press. 
Croushore, D. (2011b). Frontiers of real-time data analysis. Journal of Economic Literature, 49, $72-100$.

Croushore, D., and Stark, T. (2001). A real-time data set for macroeconomists. Journal of Econometrics, 105(1), 111-130.

Cunningham, A., Eklund, J., Jeffery, C., Kapetanios, G., and Labhard, V. (2009). A state space approach to extracting the signal from uncertain data. Journal of Business 85 Economic Statistics, 30, 173-180. doi:10.1198/jbes.2009.08171.

Faust, J., Rogers, J. H., and Wright, J. H. (2003). Exchange rate forecasting: The errors we've really made. Journal of International Economic Review, 60, 35-39.

Garratt, A., Lee, K., Mise, E., and Shields, K. (2008). Real time representations of the output gap. Review of Economics and Statistics, 90, 792-804.

Garratt, A., Lee, K., Mise, E., and Shields, K. (2009). Real time representations of the UK output gap in the presence of model uncertainty. International Journal of Forecasting, 25, 81-102.

Harvey, A. C., McKenzie, C. R., Blake, D. P. C., and Desai, M. J. (1983). Irregular data revisions. In Zellner, A. (ed.), Applied Time Series Analysis of Economic Data, pp. 329347: US Department of Commerce, Washington D.C., Economic Research Report ER-5.

Henzel, S., and Rengel, M. (2013). Dimensions of macroeconomic uncertainty: A common factor analysis. Ifo working paper series, Ifo Institute for Economic Research at the University of Munich.

Howrey, E. P. (1984). Data revisions, reconstruction and prediction: an application to inventory investment. The Review of Economics and Statistics, 66, 386-393.

Jacobs, J. P. A. M., and van Norden, S. (2011). Modeling data revisions: Measurement error and dynamics of 'true' values. Journal of Econometrics, 161, 101-109.

Jurado, K., Ludvigson, S. C., and Ng, S. (2015). Measuring Uncertainty. American Economic Review, 105(3), 1177-1216.

Kishor, N. K., and Koenig, E. F. (2012). VAR estimation and forecasting when data are subject to revision. Journal of Business and Economic Statistics, 30(2), 181-190.

Koenig, E. F., Dolmas, S., and Piger, J. (2003). The use and abuse of real-time data in economic 
forecasting. The Review of Economics and Statistics, 85(3), 618-628.

Landefeld, J. S., Seskin, E. P., and Fraumeni, B. M. (2008). Taking the pulse of the economy. Journal of Economic Perspectives, 22, 193-216.

Mankiw, N. G., and Shapiro, M. D. (1986). News or noise: An analysis of GNP revisions. Survey of Current Business (May 1986), US Department of Commerce, Bureau of Economic Analysis, 20-25.

Orphanides, A. (2001). Monetary policy rules based on real-time data. American Economic Review, 91(4), 964-985.

Orphanides, A., and van Norden, S. (2005). The reliability of inflation forecasts based on output gaps in real time. Journal of Money, Credit and Banking, 37, 583-601.

Patterson, K. D. (1995). An integrated model of the data measurement and data generation processes with an application to consumers' expenditure. Economic Journal, 105, 54-76.

Patterson, K. D. (2003). Exploiting information in vintages of time-series data. International Journal of Forecasting, 19, 177-197.

Robertson, J. C., and Tallman, E. W. (1998). Data vintages and measuring forecast model performance. Federal Reserve Bank of Atlanta Economic Review, Fourth Quarter, 4-20.

Thombs, L. A., and Schucany, W. R. (1990). Bootstrap prediction intervals for autoregression. Journal of the American Statistical Association, 85, 486-492. 


\section{Acknowledgments}

The helpful comments and suggestions of two referees of this journal are gratefully acknowledged. 
Table 1: Data Description

\begin{tabular}{l|l}
\hline Mnemonic & Description \\
\hline routputq & Real GNP/GDP \\
rconq & Real Personal Consumption Expenditures Total \\
rconndq & Real Personal Consumption Expenditures: Nondurable Goods \\
rcondq & Real Personal Consumption Expenditures: Durable Goods \\
rinvbfq & Real Gross Private Domestic Investment: Non-residential \\
rinvresq & Real Gross Private Domestic Investment: Residential \\
rexq & Real Exports of Goods and Services \\
rimpq & Real Exports of Goods and Services \\
rgq & Real Government Consumption and Gross Investment: Total \\
ruc & Unemployment Rate \\
pq & Price Index for GNP/GDP \\
pconq & Price Index for Personal Consumption Expenditures \\
pimpq & Price Index for Imports of Goods and Services \\
noutputq & Nominal GNP/GDP \\
nconq & Nominal Personal Consumption Expenditures \\
wsdq & Wage and Salary Disbursements \\
oliq & Other Labor Income \\
propiq & Proprietors' Income \\
divq & Dividends \\
pintiq & Personal Interest Income \\
tranrq & Transfer Payments \\
sscontrq & Personal Contributions for Social Insurance \\
npiq & Nominal Personal Income \\
ptaxq & Personal Tax and Nontax Payments \\
ndpiq & Nominal Disposable Personal Income \\
\hline
\end{tabular}

Source: The Real-Time Data Set for Macroeconomists (RTDSM), http://www.philadelphiafed.org/research-and-data/real-time-center/real-time-data/, see Croushore and Stark (2001). 
Table 2: RTV and EOS Box-Jenkins Prediction Intervals Coverage Rates for RTV and EOS

\begin{tabular}{|c|c|c|c|c|c|c|c|c|c|}
\hline & \multirow{2}{*}{$\begin{array}{l}\text { Ratio RTV } \\
\text { to EOS sd. }\end{array}$} & \multirow{2}{*}{$\begin{array}{r}t \text { - stat } \\
\text { for 'news' }\end{array}$} & \multirow{2}{*}{$\begin{array}{r}t \text { - stat } \\
\text { for 'noise' }\end{array}$} & \multicolumn{2}{|c|}{$50 \%$ interval } & \multicolumn{2}{|c|}{$75 \%$ interval } & \multicolumn{2}{|c|}{$90 \%$ interval } \\
\hline & & & & RTV & EOS & RTV & EOS & RTV & EOS \\
\hline routputq & 0.87 & 2.19 & -4.23 & 0.50 & 0.52 & 0.72 & 0.78 & 0.86 & 0.93 \\
\hline rconq & 1.14 & 6.29 & -0.16 & 0.52 & 0.48 & 0.74 & 0.66 & 0.84 & 0.88 \\
\hline rconndq & 1.27 & 9.46 & -0.13 & 0.48 & 0.40 & 0.72 & 0.60 & 0.86 & 0.79 \\
\hline rcondq & 1.07 & 6.44 & 1.52 & 0.52 & 0.45 & 0.69 & 0.62 & 0.83 & 0.74 \\
\hline rinvbfq & 1.18 & 2.11 & -4.53 & 0.55 & 0.48 & 0.76 & 0.64 & 0.90 & 0.86 \\
\hline rinvresq & 1.14 & 2.17 & -3.15 & 0.52 & 0.43 & 0.71 & 0.66 & 0.84 & 0.78 \\
\hline rexq & 1.04 & 3.84 & -3.32 & 0.52 & 0.36 & 0.72 & 0.67 & 0.86 & 0.84 \\
\hline rimpq & 1.13 & 5.72 & -3.20 & 0.43 & 0.38 & 0.62 & 0.60 & 0.84 & 0.72 \\
\hline rgq & 1.12 & 7.50 & -1.74 & 0.66 & 0.59 & 0.81 & 0.78 & 0.91 & 0.91 \\
\hline ruc & 1.22 & 0.49 & -0.12 & 0.50 & 0.41 & 0.69 & 0.60 & 0.79 & 0.71 \\
\hline $\mathrm{pq}$ & 1.17 & 2.60 & -1.51 & 0.52 & 0.41 & 0.72 & 0.66 & 0.90 & 0.79 \\
\hline pconq & 1.18 & 3.79 & 0.87 & 0.45 & 0.40 & 0.67 & 0.59 & 0.83 & 0.79 \\
\hline pimpq & 1.10 & 3.23 & -2.68 & 0.43 & 0.41 & 0.78 & 0.71 & 0.86 & 0.81 \\
\hline noutputq & 0.96 & 0.76 & -5.43 & 0.55 & 0.57 & 0.76 & 0.74 & 0.90 & 0.91 \\
\hline nconq & 1.13 & 4.91 & -0.67 & 0.62 & 0.57 & 0.86 & 0.74 & 0.91 & 0.88 \\
\hline wsdq & 0.46 & 1.29 & -10.13 & 0.64 & 0.81 & 0.81 & 0.91 & 0.90 & 0.98 \\
\hline oliq & 0.76 & 1.63 & -12.21 & 0.62 & 0.71 & 0.83 & 0.88 & 0.88 & 0.95 \\
\hline propiq & 1.13 & 4.28 & -5.74 & 0.62 & 0.69 & 0.86 & 0.83 & 0.95 & 0.90 \\
\hline divq & 0.69 & 1.76 & -7.44 & 0.76 & 0.86 & 0.81 & 0.90 & 0.88 & 0.93 \\
\hline pintiq & 0.62 & 0.78 & -9.13 & 0.47 & 0.66 & 0.64 & 0.81 & 0.78 & 0.93 \\
\hline tranrq & 0.86 & 1.31 & -2.17 & 0.59 & 0.55 & 0.76 & 0.79 & 0.83 & 0.86 \\
\hline sscontrq & 1.08 & 1.51 & -1.72 & 0.60 & 0.62 & 0.74 & 0.79 & 0.86 & 0.84 \\
\hline npiq & 0.69 & 0.63 & -7.81 & 0.59 & 0.74 & 0.78 & 0.84 & 0.86 & 0.91 \\
\hline ptaxq & 0.78 & -0.84 & -5.59 & 0.59 & 0.71 & 0.83 & 0.84 & 0.86 & 0.88 \\
\hline ndpiq & 0.83 & 1.37 & -5.97 & 0.59 & 0.67 & 0.83 & 0.88 & 0.90 & 0.91 \\
\hline
\end{tabular}

The second column reports the ratio of the estimated in-sample standard errors for RTV to EOS. (The EOS standard deviation is the average of the standard deviations calculated for each of the 58 rolling window estimation samples. The RTV sd. is calculated similarly). The third and fourth columns record the $t$-statistics for tests that the 1st-estimate to 15th-estimate revisions are news and noise, respectively. These tests are run once for each variable and relate to observation periods 1970:Q2 to 2007:Q2. The remaining columns record coverage rates for nominal coverages of $50 \%, 75 \%$ and $90 \%$.

For both AR and EOS the model is an $\operatorname{AR}(2)$, estimated on forecasts (vintage) origins 1996:Q2 to 2011:Q1, using a rolling window of observations (and an initial window estimated on post 1984 observations). We omit the 1999:Q4 and 2009:Q3 vintages which contain missing values for many of the series.

For the Bureau of National Accounts variables the RTDSM contains missing values for the 1996:Q1 estimates of 1995:Q4. These do not affect the EOS forecasts, because the first forecast origin is 1996:Q2, but these missing values do affect all RTV forecasts. We have simply set the values for 1995:Q4 to the 1995:Q3 values in the same (1996:Q1) vintage. 


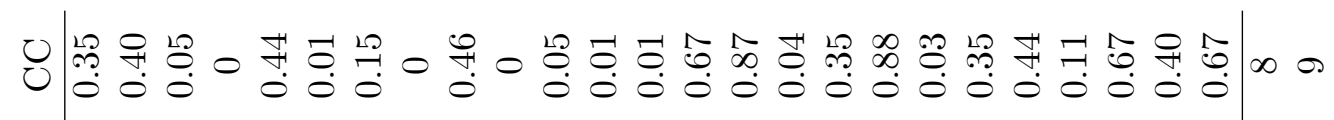

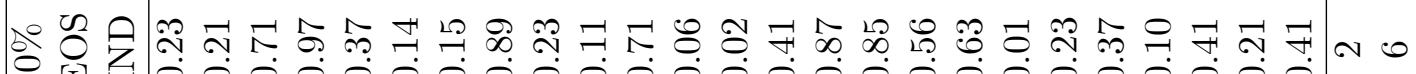
ه U

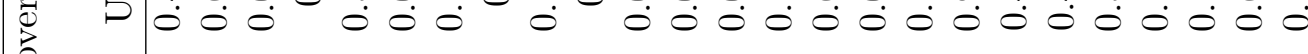

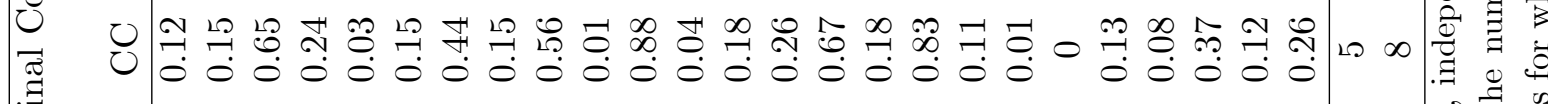

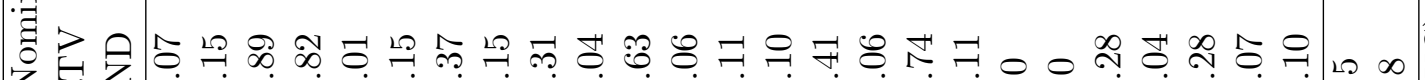

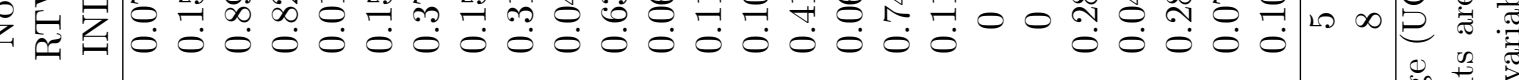

뉵

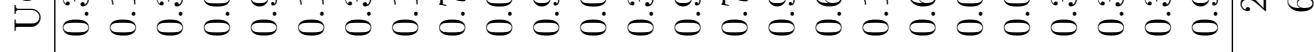

U

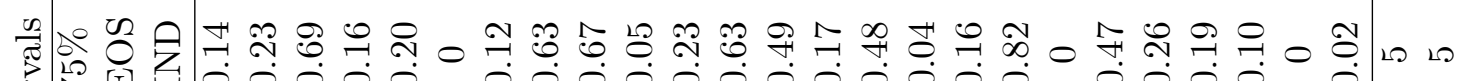

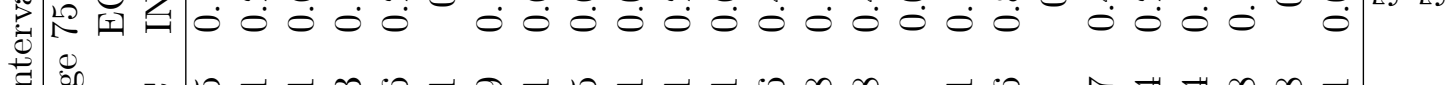

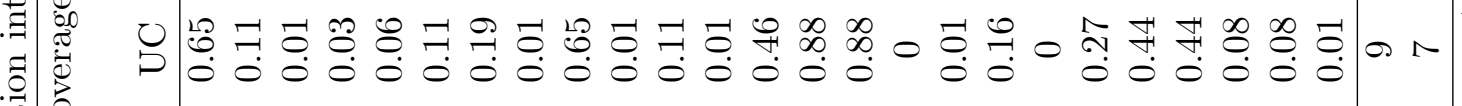

U U

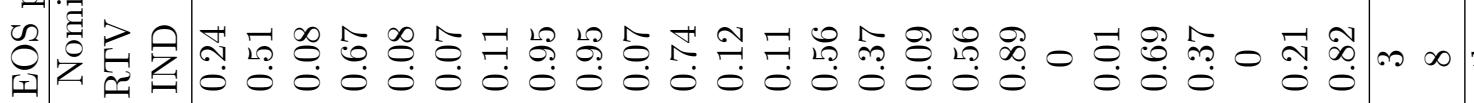

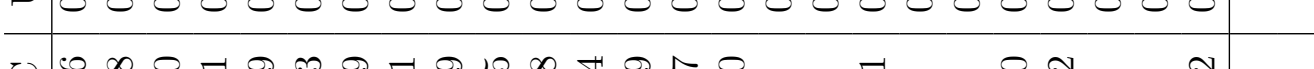

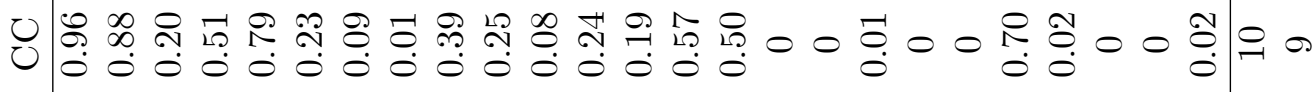

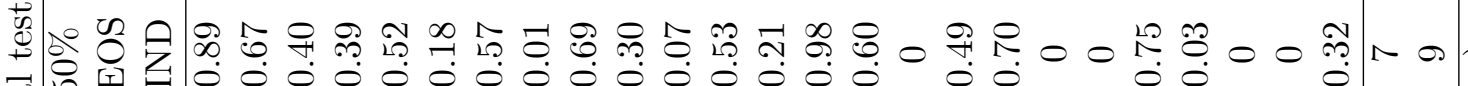
芴金任

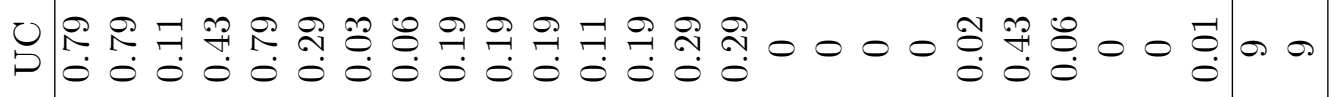

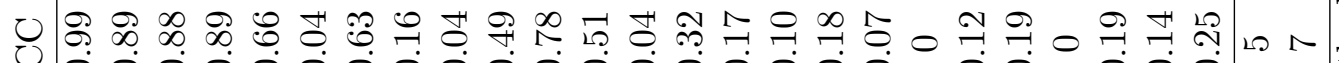
U

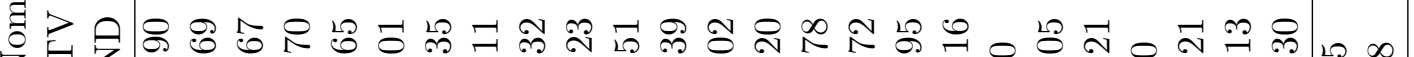

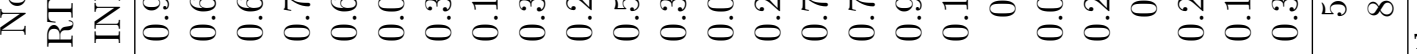

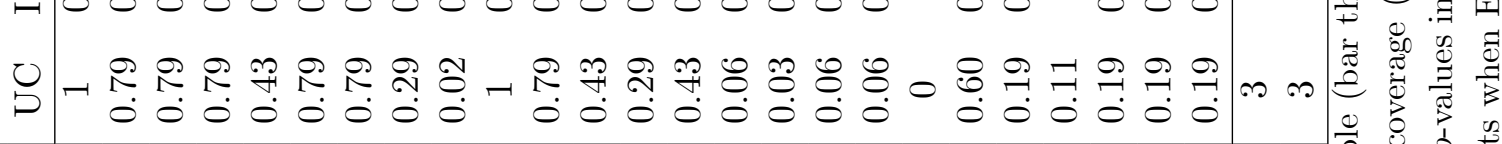


Table 4: RTV and EOS forecasts with AR models and ADL

\begin{tabular}{l|c|ccccc}
\hline & RMSFE & \multicolumn{5}{|c}{ Ratio of RMSFE of RTV to EOS } \\
\hline \hline & $\mathrm{AR}$ & $\mathrm{AR}$ & $\mathrm{ADL}_{\text {med }}$ & $\mathrm{ADL}_{\text {mea }}$ & $\mathrm{ADL}_{\text {min }}$ & $\mathrm{ADL}_{\text {max }}$ \\
\hline routputq & 0.53 & 0.95 & 0.98 & 0.98 & 0.90 & 1.09 \\
rconq & 0.52 & 1.07 & 1.08 & 1.07 & 0.97 & 1.14 \\
rconndq & 0.85 & 1.03 & 1.03 & 1.04 & 0.99 & 1.14 \\
rcondq & 2.90 & 1 & 1.01 & 1.01 & 0.97 & 1.07 \\
rinvbfq & 2.49 & 1 & 1.02 & 1.03 & 0.96 & 1.11 \\
rinvresq & 3.30 & 0.95 & 0.96 & 0.97 & 0.93 & 1.11 \\
rexq & 2.48 & 0.98 & 0.98 & 0.99 & 0.94 & 1.10 \\
rimpq & 2.56 & 1.01 & 1.03 & 1.03 & 0.94 & 1.12 \\
rgq & 0.75 & 1.02 & 1.05 & 1.08 & 1 & 1.63 \\
ruc & 0.29 & 1.03 & 1.04 & 1.05 & 1 & 1.12 \\
pq & 0.25 & 0.99 & 1 & 1.02 & 0.92 & 1.40 \\
pconq & 0.44 & 1 & 1 & 1.01 & 0.97 & 1.09 \\
pimpq & 2.51 & 1.03 & 1.04 & 1.05 & 1 & 1.14 \\
noutputq & 0.55 & 0.96 & 0.98 & 0.99 & 0.93 & 1.16 \\
nconq & 0.69 & 0.97 & 1 & 1 & 0.96 & 1.07 \\
wsdq & 0.57 & 0.78 & 0.78 & 0.78 & 0.62 & 0.92 \\
oliq & 0.50 & 0.98 & 0.96 & 0.96 & 0.85 & 1.16 \\
propiq & 1.22 & 1.13 & 1.14 & 1.17 & 1.07 & 1.77 \\
divq & 7.09 & 1.32 & 1.36 & 1.36 & 1.25 & 1.46 \\
pintiq & 1.29 & 1 & 0.96 & 0.95 & 0.82 & 1.11 \\
tranrq & 1.66 & 0.98 & 0.98 & 0.99 & 0.92 & 1.06 \\
sscontrq & 1.56 & 1 & 1.01 & 1.01 & 0.96 & 1.06 \\
npiq & 0.54 & 0.99 & 1 & 1 & 0.81 & 1.10 \\
ptaxq & 4.94 & 1.03 & 1.04 & 1.04 & 0.95 & 1.15 \\
ndpiq & 0.81 & 1.09 & 1.09 & 1.09 & 0.96 & 1.31 \\
\hline
\end{tabular}

Notes. The table reports the RMSEs for a rolling forecasting scheme, with initial window beginning in 1984:Q1. Out-of-sample forecast period is 1996:Q2 to 2011:Q1. The actual values used to calculate forecast errors are first-release values. 
Table 5: Monte Carlo of small-sample coverage rates of EOS and RTV intervals

\begin{tabular}{|c|c|c|c|c|c|c|}
\hline & \multicolumn{2}{|c|}{$50 \%$} & \multicolumn{2}{|c|}{$75 \%$} & \multicolumn{2}{|c|}{$90 \%$} \\
\hline & EOS & RTV & EOS & RTV & EOS & RTV \\
\hline \multicolumn{7}{|c|}{ Jacobs and van Norden (2011) parameter values } \\
\hline \multicolumn{7}{|c|}{ A. News, no spillovers } \\
\hline 15 & 0.45 & 0.43 & 0.68 & 0.66 & 0.83 & 0.81 \\
\hline 25 & 0.49 & 0.47 & 0.73 & 0.71 & 0.88 & 0.86 \\
\hline 50 & 0.51 & 0.48 & 0.76 & 0.73 & 0.91 & 0.88 \\
\hline 200 & 0.53 & 0.50 & 0.78 & 0.75 & 0.92 & 0.90 \\
\hline \multicolumn{7}{|c|}{ B. Noise, no spillovers } \\
\hline 15 & 0.43 & 0.43 & 0.65 & 0.66 & 0.81 & 0.81 \\
\hline 25 & 0.46 & 0.46 & 0.70 & 0.70 & 0.85 & 0.86 \\
\hline 50 & 0.47 & 0.48 & 0.72 & 0.73 & 0.88 & 0.88 \\
\hline 200 & 0.49 & 0.50 & 0.74 & 0.75 & 0.89 & 0.90 \\
\hline \multicolumn{7}{|c|}{ C. News \& spillovers } \\
\hline 15 & 0.46 & 0.43 & 0.70 & 0.66 & 0.84 & 0.81 \\
\hline 25 & 0.51 & 0.47 & 0.75 & 0.71 & 0.89 & 0.86 \\
\hline 50 & 0.53 & 0.49 & 0.78 & 0.73 & 0.92 & 0.88 \\
\hline 200 & 0.54 & 0.50 & 0.79 & 0.75 & 0.93 & 0.90 \\
\hline \multicolumn{7}{|c|}{ D. Noise \& spillovers } \\
\hline 15 & 0.43 & 0.43 & 0.65 & 0.66 & 0.81 & 0.81 \\
\hline 25 & 0.45 & 0.46 & 0.70 & 0.70 & 0.85 & 0.86 \\
\hline 50 & 0.47 & 0.48 & 0.72 & 0.73 & 0.87 & 0.88 \\
\hline 200 & 0.49 & 0.50 & 0.74 & 0.75 & 0.89 & 0.90 \\
\hline \multicolumn{7}{|c|}{ More prominent data revisions } \\
\hline \multicolumn{7}{|c|}{ A. News, no spillovers } \\
\hline 15 & 0.46 & 0.42 & 0.69 & 0.64 & 0.84 & 0.79 \\
\hline 25 & 0.52 & 0.47 & 0.76 & 0.70 & 0.90 & 0.86 \\
\hline 50 & 0.54 & 0.48 & 0.79 & 0.73 & 0.92 & 0.88 \\
\hline 200 & 0.56 & 0.50 & 0.80 & 0.75 & 0.94 & 0.89 \\
\hline \multicolumn{7}{|c|}{ B. Noise, no spillovers } \\
\hline 15 & 0.38 & 0.43 & 0.59 & 0.65 & 0.75 & 0.81 \\
\hline 25 & 0.40 & 0.47 & 0.63 & 0.71 & 0.79 & 0.86 \\
\hline 50 & 0.41 & 0.48 & 0.65 & 0.73 & 0.81 & 0.89 \\
\hline 200 & 0.43 & 0.50 & 0.66 & 0.75 & 0.82 & 0.89 \\
\hline \multicolumn{7}{|c|}{ C. News \& spillovers } \\
\hline 15 & 0.48 & 0.42 & 0.71 & 0.64 & 0.85 & 0.80 \\
\hline 25 & 0.53 & 0.47 & 0.77 & 0.70 & 0.91 & 0.86 \\
\hline 50 & 0.55 & 0.48 & 0.80 & 0.73 & 0.93 & 0.88 \\
\hline 200 & 0.57 & 0.50 & 0.82 & 0.74 & 0.94 & 0.89 \\
\hline \multicolumn{7}{|c|}{ D. Noise \& spillovers } \\
\hline 15 & 0.37 & 0.43 & 0.58 & 0.65 & 0.74 & 0.81 \\
\hline 25 & 0.40 & 0.47 & 0.62 & 0.71 & 0.79 & 0.86 \\
\hline 50 & 0.41 & 0.48 & 0.64 & 0.73 & 0.81 & 0.89 \\
\hline 200 & 0.42 & 0.50 & 0.65 & 0.75 & 0.82 & 0.89 \\
\hline
\end{tabular}

'More prominent data revisions' refers to the case where the standard deviation of the disturbance term in the data generating process is multiplied by 0.25 , relative to the Jacobs and van Norden (2011) estimated value.

When there are no revisions, the coverage rates are: $0.43,0.46,0.48,0.50$ (for a nominal $50 \%$, for estimation samples 15 to 200); 0.66, 0.70, 0.73, 0.75 (for a nominal 75\%); and 0.82, 0.86, $0.88,0.89$ (for a nominal $90 \%$ ). 
Table 6: Monte Carlo of small-sample coverage rates of RTV, EOS and adapted RTV intervals for revised values

\begin{tabular}{|c|c|c|c|c|c|c|c|c|c|}
\hline & \multicolumn{3}{|c|}{$50 \%$} & \multicolumn{3}{|c|}{$75 \%$} & \multicolumn{3}{|c|}{$90 \%$} \\
\hline & RTV & EOS & $\operatorname{RTV}(\mathrm{n}=3)$ & RTV & EOS & $\operatorname{RTV}(\mathrm{n}=3)$ & RTV & EOS & $\operatorname{RTV}(\mathrm{n}=3)$ \\
\hline \multicolumn{10}{|c|}{ More prominent data revisions } \\
\hline \multicolumn{10}{|c|}{ A. News, no spillovers } \\
\hline 15 & 0.39 & 0.43 & 0.34 & 0.60 & 0.66 & 0.52 & 0.76 & 0.81 & 0.66 \\
\hline 25 & 0.42 & 0.47 & 0.44 & 0.65 & 0.70 & 0.67 & 0.81 & 0.86 & 0.83 \\
\hline 50 & 0.43 & 0.48 & 0.47 & 0.67 & 0.73 & 0.72 & 0.83 & 0.88 & 0.88 \\
\hline 200 & 0.45 & 0.50 & 0.50 & 0.68 & 0.75 & 0.74 & 0.85 & 0.90 & 0.89 \\
\hline \multicolumn{10}{|c|}{ B. Noise, no spillovers } \\
\hline 15 & 0.49 & 0.43 & 0.33 & 0.72 & 0.65 & 0.52 & 0.86 & 0.81 & 0.66 \\
\hline 25 & 0.53 & 0.46 & 0.44 & 0.77 & 0.70 & 0.67 & 0.91 & 0.86 & 0.83 \\
\hline 50 & 0.55 & 0.47 & 0.48 & 0.80 & 0.72 & 0.72 & 0.93 & 0.88 & 0.88 \\
\hline 200 & 0.57 & 0.49 & 0.50 & 0.82 & 0.74 & 0.74 & 0.94 & 0.89 & 0.90 \\
\hline \multicolumn{10}{|c|}{ C. News \& spillovers } \\
\hline 15 & 0.38 & 0.44 & 0.34 & 0.59 & 0.66 & 0.53 & 0.74 & 0.81 & 0.66 \\
\hline 25 & 0.41 & 0.47 & 0.44 & 0.63 & 0.70 & 0.67 & 0.80 & 0.86 & 0.83 \\
\hline 50 & 0.42 & 0.48 & 0.47 & 0.65 & 0.73 & 0.72 & 0.82 & 0.88 & 0.88 \\
\hline 200 & 0.43 & 0.50 & 0.50 & 0.67 & 0.75 & 0.74 & 0.83 & 0.90 & 0.89 \\
\hline \multicolumn{10}{|c|}{ D. Noise \& spillovers } \\
\hline 15 & 0.49 & 0.43 & 0.34 & 0.72 & 0.65 & 0.52 & 0.86 & 0.81 & 0.66 \\
\hline 25 & 0.53 & 0.46 & 0.44 & 0.77 & 0.70 & 0.67 & 0.91 & 0.86 & 0.83 \\
\hline 50 & 0.56 & 0.47 & 0.48 & 0.81 & 0.72 & 0.72 & 0.93 & 0.88 & 0.88 \\
\hline 200 & 0.57 & 0.49 & 0.50 & 0.82 & 0.74 & 0.74 & 0.95 & 0.89 & 0.90 \\
\hline \multicolumn{10}{|c|}{ More prominent data revisions And Greater Persistence } \\
\hline \multicolumn{10}{|c|}{ A. News, no spillovers } \\
\hline 15 & 0.40 & 0.41 & 0.28 & 0.62 & 0.62 & 0.44 & 0.78 & 0.78 & 0.57 \\
\hline 25 & 0.45 & 0.45 & 0.40 & 0.69 & 0.69 & 0.62 & 0.84 & 0.84 & 0.78 \\
\hline 50 & 0.47 & 0.47 & 0.47 & 0.72 & 0.72 & 0.71 & 0.87 & 0.88 & 0.86 \\
\hline 200 & 0.49 & 0.50 & 0.50 & 0.74 & 0.74 & 0.75 & 0.89 & 0.89 & 0.90 \\
\hline \multicolumn{10}{|c|}{ B. Noise, no spillovers } \\
\hline 15 & 0.44 & 0.39 & 0.26 & 0.67 & 0.60 & 0.41 & 0.82 & 0.76 & 0.55 \\
\hline 25 & 0.48 & 0.42 & 0.37 & 0.73 & 0.65 & 0.59 & 0.88 & 0.82 & 0.75 \\
\hline 50 & 0.52 & 0.44 & 0.44 & 0.77 & 0.68 & 0.68 & 0.91 & 0.84 & 0.84 \\
\hline 200 & 0.56 & 0.46 & 0.49 & 0.81 & 0.70 & 0.74 & 0.94 & 0.87 & 0.89 \\
\hline \multicolumn{10}{|c|}{ C. News \& spillovers } \\
\hline 15 & 0.40 & 0.41 & 0.28 & 0.62 & 0.62 & 0.44 & 0.78 & 0.78 & 0.58 \\
\hline 25 & 0.44 & 0.45 & 0.40 & 0.68 & 0.69 & 0.62 & 0.84 & 0.84 & 0.78 \\
\hline 50 & 0.47 & 0.47 & 0.47 & 0.71 & 0.72 & 0.71 & 0.87 & 0.88 & 0.86 \\
\hline 200 & 0.49 & 0.50 & 0.50 & 0.74 & 0.75 & 0.75 & 0.89 & 0.90 & 0.90 \\
\hline \multicolumn{10}{|c|}{ D. Noise \& spillovers } \\
\hline 15 & 0.44 & 0.39 & 0.26 & 0.67 & 0.60 & 0.42 & 0.82 & 0.77 & 0.55 \\
\hline 25 & 0.48 & 0.42 & 0.37 & 0.73 & 0.65 & 0.59 & 0.88 & 0.82 & 0.75 \\
\hline 50 & 0.53 & 0.44 & 0.44 & 0.78 & 0.68 & 0.68 & 0.92 & 0.84 & 0.84 \\
\hline 200 & 0.56 & 0.46 & 0.49 & 0.82 & $035_{1}$ & 0.74 & 0.94 & 0.87 & 0.89 \\
\hline
\end{tabular}

The first half of the table 'More prominent data revisions' refers to the case where the standard deviation of the disturbance term in the data generating process is multiplied by 0.25 , relative to the Jacobs and van Norden (2011) estimated value. In the second half of the table in addition there is 'Greater persistence': the autoregressive parameters of the true process are set to 0.90 and 0.05 . 


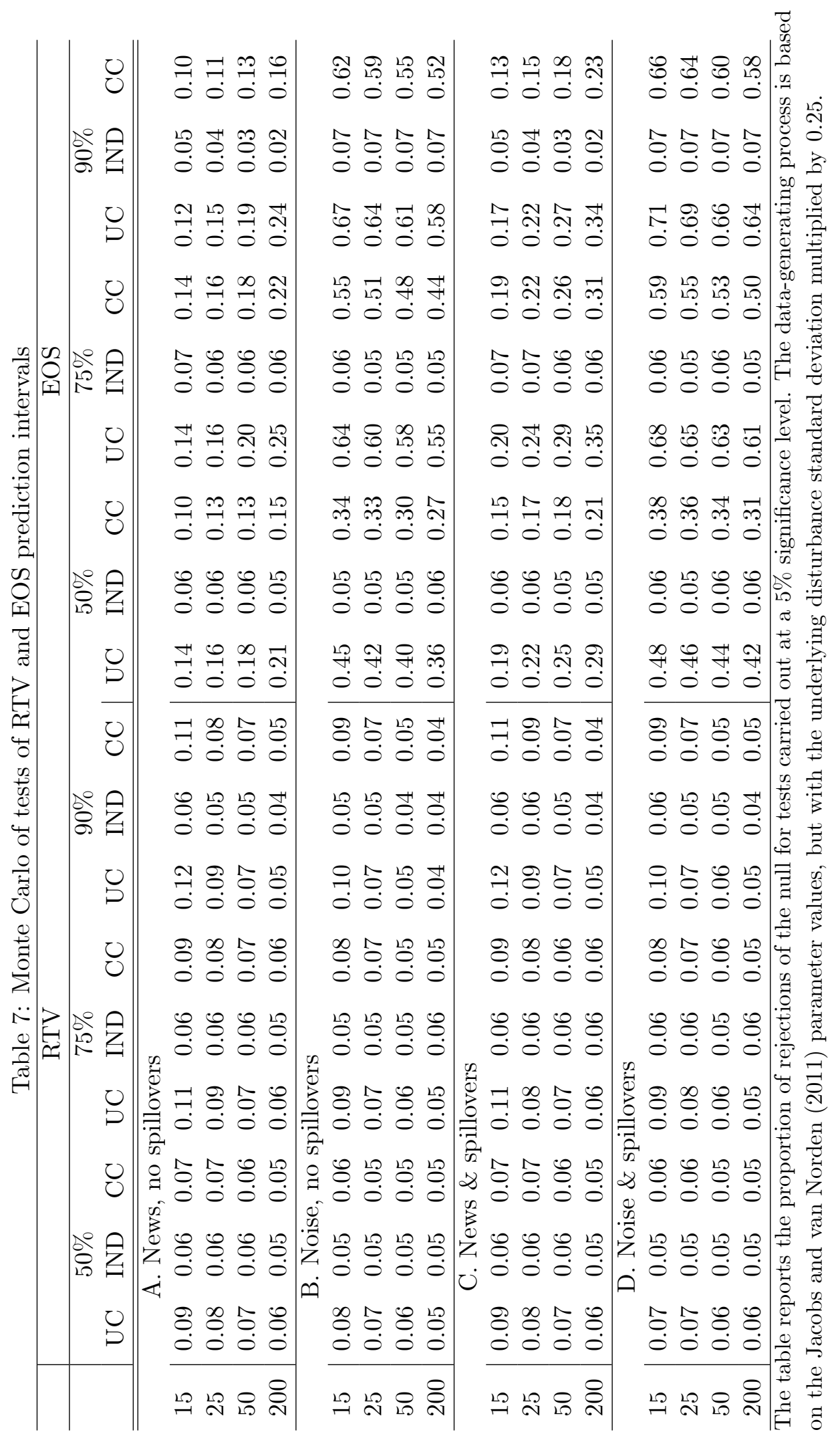

